\title{
Some remarks on the level functions and their applications
}

\author{
Paweł Foralewski, Karol Leśnik, and Lech Maligranda
}

Summary. A comparison of the level functions considered by Halperin and Sinnamon is discussed. Moreover, connections between Lorentz-type spaces, down spaces, Cesàro spaces, and Sawyer's duality formula are explained. Applying Sinnamon's ideas, we prove the duality theorem for Orlicz-Lorentz spaces which generalizes a recent result by Kamińska, Leśnik, and Raynaud (and Nakamura). Finally, some applications of the level functions to the geometry of Orlicz-Lorentz spaces are presented.

Received: 2016-04-20, Accepted: 2016-06-18
Keywords level functions; dual spaces; Orlicz-Lorentz spaces; Halperin spaces; Cesàro spaces

MSC 2010

46E30; 46B20; 46B42

Dedicated to Professor Henryk Hudzik on the occasion of his 70 th birthday.

\section{Introduction}

The classical Lorentz spaces $\Lambda_{p, w}$ consist of all equivalence classes of functions $f \in L^{0}$ such that

$$
\|f\|_{p, w}=\left(\int_{I} f^{*}(t)^{p} w(t) d t\right)^{1 / p}<\infty,
$$

where $1 \leqslant p<\infty$ and $w$ is a locally integrable positive weight function. These spaces were defined in 1951 by Lorentz [36]. He showed that $\|\cdot\|_{p, w}$ is a norm if and only if the weight $w$ is a decreasing function ([36, Thm 1]). Moreover, he proved that for $p>1$, the spaces $\Lambda_{p, w}$

Paweł Foralewski, Faculty of Mathematics and Computer Science, Adam Mickiewicz University, Umultowska 87, 60-769 Poznań, Poland (e-mail: katon@amu.edu.pl)

Karol Leśnik, Institute of Mathematics of Electric Faculty, Poznań University of Technology, Piotrowo 3a, 60-965 Poznań, Poland (e-mail: klesnik@vp.pl)

Lech Maligranda, Department of Engineering Sciences and Mathematics, Luleå University of Technology, SE-971 87 Luleå, Sweden (e-mail: lech.maligranda@ltu.se) 
are reflexive (see [36, Thms 4, 5]; see also [37, Thm 3.7.3]). Some results on the Lorentz spaces $\Lambda_{p, w}$ with more general weights (not only decreasing) and for $0<p<\infty$ were obtained in $[7,11,20]$, and [47], which is the published version of the preprint [11].

In 1953, Halperin [12] gave an explicit description of the dual norm to the Lorentz norm. In order to do this, he introduced level intervals and level functions with respect to the weight $w$. His idea was picked up by Lorentz, who presented his point of view on level functions and gave an alternative proof of Halperin's duality formula (cf. [37]).

It happened that the methods developed by Halperin yielded at the same time a so-called $D$-type Hölder inequality (with the exact norm given in terms of the level functions). The notion of the $D$ type corresponds to the character of the proven inequality, which is a version of Hölder's inequality with only decreasing functions in one space being considered ("D" stands for "decreasing"). Namely, he proved

$$
\begin{aligned}
\sup \left\{\int_{I}|f(t)| g(t) d t: g \geqslant 0, g \text { decreasing, }\|g\|_{L_{p}(w)} \leqslant 1\right\} \\
\quad=\left(\int_{I}\left(\frac{f^{o}(t)}{w(t)}\right)^{q} w(t) d t\right)^{1 / q},
\end{aligned}
$$

where $1 / p+1 / q=1$ and $f^{o}$ is the level function of $f$ with respect to $w$.

The same idea of restricting Hölder's inequality to decreasing functions was pursued by Sawyer, who in [41] proved a very useful duality equivalence formula, in place of Halperin's description (1):

$$
\begin{array}{r}
\sup \left\{\int_{0}^{\infty}|f(t)| g(t) d t: g \geqslant 0, g \text { decreasing, }\|g\|_{L_{p}(w)} \leqslant 1\right\} \\
\quad \approx\left(\int_{0}^{\infty}\left(\frac{\int_{0}^{x}|f(t)| d t}{\int_{0}^{x} w(t) d t}\right)^{q} w(x) d x\right)^{1 / q}+\frac{\|f\|_{L_{1}}}{\left(\int_{0}^{\infty} w(t) d t\right)^{1 / p}} .
\end{array}
$$

This formula found a number of important applications in estimating operators from harmonic analysis. Notice that it is also a version of the above-mentioned $D$-type Hölder inequality, but this time the representation is not exact, i.e. there is only an equivalence of norms. Sawyer mentions Halperin's exact result (1) on page 146 but he referred only to the papers by Lorentz [36,37] and not to the original paper by Halperin [12].

One more interpretation can be found in the work of Sinnamon, who in [43] introduced a so-called down space $D\left(L_{p}(w)\right)$ defined by the norm

$$
\|f\|_{D\left(L_{p}(w)\right)}=\sup \left\{\int_{I}|f(t)| g(t) w(t) d t: g \geqslant 0, g \text { decreasing, }\|g\|_{L_{q}(w)} \leqslant 1\right\} .
$$

Sinnamon was able to find an exact description of this norm in the space $D\left(L_{p}(w)\right)$ as well as of the norm in its dual space (up to isometry). These results were then generalized 
to symmetric spaces and Banach function spaces by Sinnamon himself and many other authors $[10,13,19,21,23,30]$.

In turn, Kamińska and Mastyło [21] generalized Sawyer's formula to symmetric spaces over a general measure space. In this paper, we will survey and compare both types of generalizations, which turn out to be the same.

On the other hand, the Lorentz spaces $\Lambda_{p, w}$ and their duality was generalized as well. We will be interested in the classical generalization where the power function is replaced by an Orlicz function, leading to Orlicz-Lorentz spaces $\Lambda_{\varphi, w}$ investigated by many authors (see, e.g., $[7-9,15,17,40]$ and references therein). In [6] was posed the problem of finding an exact (isometric) description of the dual space to the Orlicz-Lorentz space $\Lambda_{\varphi, w}$, and it was solved by Kamińska, Leśnik, and Raynaud [19] in the case when $\varphi$ is an $N$-function. However, it appears that Orlicz-Lorentz spaces were already introduced by Nakamura in 1970 in his completely unknown paper [40] (this paper has zero citations in MathSciNet). Furthermore, Nakamura was even able to give a description of the dual space to the Orlicz-Lorentz space $\Lambda_{\varphi, w}$, using Halperin's ideas and taking advantage of the theory of modular spaces.

The duality of Orlicz-Lorentz spaces will be our other subject in this paper. We will give another proof of the duality and investigate the properties of the dual spaces.

The goal of the paper is twofold. First, we will survey and explain ideas behind level functions, Sawyer's formula, and down spaces, to make the subject more understandable. Second, we will apply the presented ideas to give a number of new results concerning the duality of Orlicz-Lorentz spaces and the properties of their duals.

The paper is divided in four parts. In the first part, we discuss two kinds of level functions - of Halperin's and of Lorentz-Sinnamon's. We start with some elementary introduction concerning $w$-concave functions and $w$-concave majorants. Using these notions we will define the two kinds of level functions and discuss their relations. In this part we also present a number of examples which provide an intuitive grasp of this theory and explain in detail what the level intervals and level functions are all about, to be able to use these concepts in the following sections.

The second part is devoted to Sawyer's formula, the down spaces, and their connections with Cesàro-type spaces. We discuss briefly the history of Sawyer's formula and of its generalizations. It will be shown in particular that the abstract Sawyer's formula, proved by Kamińska and Mastyło [21], is equivalent to Sawyer's formula that comes from Sinnamon's theory of down spaces.

In the third part, we turn our attention to duality of Orlicz-Lorentz spaces, where the level functions play a crucial role. We present a short proof of the duality for $\Lambda_{p, w}$ spaces which is a compilation of Halperin's and Lorentz's proof. Then we switch to general Orlicz-Lorentz spaces and show how an assumption in the duality theorem of Kamińska, 
Leśnik, and Raynaud [19] can be removed. This time the proof is based on an idea of Sinnamon's from his down spaces.

The final section contains considerations on the geometry of Orlicz-Lorentz spaces $\Lambda_{p, w}$ and their (metric) duals $\mathcal{M}_{\psi, w}$. Among other things, we prove that $\mathcal{M}_{\psi, w}$ are never strictly convex and hence that Orlicz-Lorentz spaces are mostly non-smooth. We discuss also order continuity of $\mathcal{M}_{\psi, w}$ spaces. It is worth mentioning that these "bad" geometric properties of $\mathcal{M}_{\psi, w}$ are generated by the modular $\mathcal{P}_{\psi, w}$ defining the norm in $\mathcal{M}_{\psi, w}$ and do not depend on the properties of $\psi$ and $w$ at all. These results correspond to the investigations by Levis and Cuenya [33,34] and Biktasheva [2].

We start with some notation that is common to all sections, whereas further required definitions will be recalled along the way.

In this paper, by decreasing or increasing functions we mean functions that are non-increasing or non-decreasing, respectively.

Let $I=[0, \infty)$ or $I=[0, \lambda]$ with $0<\lambda<\infty$. By $\mathcal{W}=\mathcal{W}(I)$ we denote the class of weights $w$, that is, functions on $I$, which are positive, i.e. $w(t)>0$ for $t \in I$, and locally integrable, i.e. $W(t)=\int_{0}^{t} w(s) d s<\infty$ for $t \in I$. By $\mathcal{W}_{d}=\mathcal{W}_{d}(I)$ we mean the weights from $\mathcal{W}$ which additionally are decreasing functions on $I$. Let $W(\infty)=\int_{0}^{\infty} w(s) d s$. Lebesgue measure will be denoted by $|\cdot|$. For a weight $w$ on $I$ consider the measure $\mu$ on $I$ such that $d \mu=w(t) d t$, i.e. $w$ is a Radon-Nikodym derivative of $\mu$ with respect to Lebesgue measure ( $\mu$ will always be understood in this way in the paper).

As usual, $L^{0}=L^{0}(I, \mu)$ is the space of all equivalence classes of $\mu$-measurable functions on $I$. A subspace $X$ of $L^{0}$ is called a Banach function space (B.f.s. for short) if it is a Banach space with a norm satisfying the ideal condition: $|f| \leqslant|g| \mu$-a.e., $f \in L^{0}$, and $g \in X$ imply $f \in X$ and $\|f\| \leqslant\|g\|$. We will assume that $X$ has the Fatou property, i.e. if $f_{n} \uparrow f \mu$-a.e. and $\left\|f_{n}\right\|_{X}$ is bounded, then $f \in X$ and $\left\|f_{n}\right\|_{X} \uparrow\|f\|_{X}$. The Köthe dual of a B.f.s. $X$ is defined by the norm

$$
\|f\|_{X^{\prime}}=\sup \left\{\int_{I}|f(t) g(t)| d \mu:\|g\|_{X} \leqslant 1\right\}=\sup \left\{\int_{I}|f(t) g(t)| w(t) d t:\|g\|_{X} \leqslant 1\right\} .
$$

For a given function $f \in L^{0}(I, \mu)$, its rearrangement (with respect to the measure $\mu$ ) is defined as

$$
f_{w}^{*}(t)=\inf \{\lambda>0: \mu(\{s>0:|f(s)|>\lambda\}) \leqslant t\} .
$$

A B.f.s. $X(w)$ on $(I, \mu)$ will be called $w$-symmetric whenever $f \in X(w), g \in L^{0}(I, \mu)$, and $f_{w}^{*}=g_{w}^{*}$ imply $g \in X(w)$ and $\|g\|_{X(w)}=\|f\|_{X(w)}$.

Notice that $\mu$ is the Lebesgue measure if $w \equiv 1$, and we will distinguish this case by writing no index " $w$ " in the above definitions. Similarly, we write $X(w)$ to emphasize that the underlying measure space is $(I, \mu)$ with $d \mu=w(t) d t$. 
We say that a symmetric space $X$ (over Lebesgue measure) is the canonical (Luxemburg) representation of the $w$-symmetric space $X(w)$ whenever

$$
\|f\|_{X(w)}=\left\|f_{w}^{*}\right\|_{X} .
$$

Note that

$$
\|f\|_{X(w)}=\left\|f_{w}^{*}\right\|_{X}=\left\|f\left(W^{-1}\right)\right\|_{X} .
$$

(cf. [38, Proposition 1]).

For more information, including the classical results concerning Banach function spaces and symmetric spaces, we refer to the books $[1,26,35,49]$.

We say that $f$ is submajorized by $g$ and write $f<_{w} g$ if

$$
\int_{0}^{t} f_{w}^{*}(s) w(s) d s \leqslant \int_{0}^{t} g_{w}^{*}(s) w(s) d s \quad \text { for all } t \in I .
$$

We will just write $f<g$ if $w \equiv 1$.

\section{Level functions of Halperin's and of Sinnamon's, and their comparison}

This section is devoted to the level functions that Halperin [12] and Lorentz [37] introduced in order to describe duality of the Lorentz-type spaces. To find a common "root" and present the ideas clearly, we will restate some known results concerning generalized concave majorants. In the case of the interval $[0,1]$ this theory has already been described by Lorentz in [37], and later by Annika Haaker (Sparr) in [11]. Sinnamon generalized it to the case of $\mathbb{R}$ with a general Borel measure $([43,44])$. We are going to use the level functions in the context of Lorentz spaces and, therefore, we focus on the case of an interval or the semiaxis $I$ (with the absolutely continuous measure $\mu$, as explained in the introduction). We should mention that the notion of concave majorants appears also in an isometric description of duality of Cesàro spaces (see [18]).

Let $w \in \mathcal{W}$. Recall that a non-negative function $L$ on $I$ is said to be $w$-affine if it is of the form

$$
L(t)=c W(t)+d
$$

for some real constants $c, d$. We will say that an increasing positive function $F$ on $I$ is $w$-concave if, for any $0 \leqslant a<b \in I$ and any $w$-affine function $L$ satisfying $F(a)=L(a)$, $F(b)=L(b)$, we have

$$
L(t) \leqslant F(t) \text { for every } t \in(a, b) .
$$

We start with the following theorem formulated first by Lorentz in the case of $[0,1]$ (see [37, pages 68-70]; see also [43, Thm 2.7] and [47, Lemma 4.4]).

2.1. Theorem. Let $F$ be an increasing positive function on $I$, and let $w \in \mathcal{W}$. The following statements are equivalent. 
(i) The function $F$ is $w$-concave.

(ii) For all $0 \leqslant a<t<b \in I$,

$$
\frac{F(t)-F(a)}{W(t)-W(a)} \geqslant \frac{F(b)-F(a)}{W(b)-W(a)} .
$$

Moreover, if $F(t)=\int_{0}^{t} f(s) d s$, where $f$ is a non-negative locally integrable function on $I$, the above conditions are equivalent to the following condition:

(iii) The function $f$ is of the form $f(t)=D(t) w(t)$, where $D$ is a decreasing function.

2.2. Remark. The inequality (3) can be formulated equivalently as

$$
\frac{F(b)-F(a)}{W(b)-W(a)} \geqslant \frac{F(b)-F(t)}{W(b)-W(t)}
$$

or

$$
\frac{F(t)-F(a)}{W(t)-W(a)} \geqslant \frac{F(b)-F(t)}{W(b)-W(t)}
$$

Indeed, it is easy to see that for all positive real numbers $v, x, y$, and $z$ the following inequalities are equivalent:

$$
\frac{v}{y} \geqslant \frac{v+x}{y+z}, \quad \frac{v+x}{y+z} \geqslant \frac{x}{z}, \quad \text { and } \quad \frac{v}{y} \geqslant \frac{x}{z} .
$$

Then, denoting $v=F(t)-F(a), x=F(b)-F(t), y=W(t)-W(a)$, and $z=W(b)-W(t)$, we immediately get that inequalities (3), (4), and (5) are equivalent. Moreover, combining the above inequalities, we get

$$
\frac{F\left(b_{1}\right)-F\left(a_{1}\right)}{W\left(b_{1}\right)-W\left(a_{1}\right)} \geqslant \frac{F\left(b_{2}\right)-F\left(a_{2}\right)}{W\left(b_{2}\right)-W\left(a_{2}\right)}
$$

for all $a_{1}, b_{1}, a_{2}$, and $b_{2}$ such that $0 \leqslant a_{1}<b_{1} \in I, 0 \leqslant a_{2}<b_{2} \in I, a_{1} \leqslant a_{2}$, and $b_{1} \leqslant b_{2}$.

Proof of Theorem 2.1. (i) $\Rightarrow$ (ii). Fix $0 \leqslant a<t_{0}<b \in I$. Then for the $w$-affine function $L$ defined by the formula

$$
L(t)=\frac{F(b)-F(a)}{W(b)-W(a)} W(t)+F(a)-\frac{F(b)-F(a)}{W(b)-W(a)} W(a),
$$

we have $L(a)=F(a)$ and $L(b)=F(b)$. Analogously, the function

$$
L_{t_{0}}(t)=\frac{F\left(t_{0}\right)-F(a)}{W\left(t_{0}\right)-W(a)} W(t)+F(a)-\frac{F\left(t_{0}\right)-F(a)}{W\left(t_{0}\right)-W(a)} W(a)
$$

is $w$-affine, $L_{t_{0}}(a)=F(a)$, and $L_{t_{0}}\left(t_{0}\right)=F\left(t_{0}\right)$. Then, by $w$-concavity of $F$, we have

$$
L\left(t_{0}\right) \leqslant F\left(t_{0}\right)=L_{t_{0}}\left(t_{0}\right),
$$


and so

$$
\frac{F\left(t_{0}\right)-F(a)}{W\left(t_{0}\right)-W(a)} \geqslant \frac{F(b)-F(a)}{W(b)-W(a)} .
$$

(ii) $\Rightarrow$ (i). Now let $0 \leqslant a<b \in I$ be arbitrary, and choose a $w$-affine function $L$ satisfying $L(a)=F(a), L(b)=F(b)$. It is easy to see that $L$ must be of the form (8). Let us fix some $t_{0} \in(a, b)$. Then for the function $L_{t_{0}}$, defined as in (9), we have $L_{t_{0}}(a)=F(a)$ and $L_{t_{0}}\left(t_{0}\right)=F\left(t_{0}\right)$. Hence by inequality (3)

$$
L\left(t_{0}\right) \leqslant L_{t_{0}}\left(t_{0}\right)=F\left(t_{0}\right),
$$

and we get that $F$ is $w$-concave, since $t_{0}$ was arbitrary.

(ii) $\Rightarrow$ (iii). By inequality (7), the function

$$
D_{h}(t)=\frac{F(t+h)-F(t)}{W(t+h)-W(t)}=\frac{h^{-1} \int_{t}^{t+h} f(s) d s}{h^{-1} \int_{t}^{t+h} w(s) d s}
$$

is a decreasing function of $t$ for every positive $h$. Therefore, by the Lebesgue theorem, the limit

$$
D(t)=\lim _{h \rightarrow 0^{+}} D_{h}(t)=\frac{f(t)}{w(t)},
$$

exists for almost every $t$ and $D$ is decreasing (up to a set of measure zero), which gives (iii). (iii) $\Rightarrow$ (ii). Finally assume that $f(t)=D(t) w(t)$, where $\mathrm{D}$ is a decreasing function. Then, for $0 \leqslant a<b \in I$ and $t \in(a, b)$,

$$
\frac{F(t)-F(a)}{W(t)-W(a)}=\frac{\int_{a}^{t} D(s) w(s) d s}{\int_{a}^{t} w(s) d s} \geqslant D(t) \geqslant \frac{\int_{t}^{b} D(s) w(s) d s}{\int_{t}^{b} w(s) d s}=\frac{F(b)-F(t)}{W(b)-W(t)},
$$

and by Remark 2.2 we obtain (ii).

2.3. Theorem. Let $w \in \mathcal{W}$. If $F$ is an increasing $w$-concave function such that $\lim _{t \rightarrow 0^{+}} F(t)=$ $F(0)=0$, then $F$ is absolutely continuous on $I$.

Proof. We need to show that for any $\varepsilon>0$ there exists $\delta>0$ such that whenever a finite sequence of pairwise disjoint subintervals $\left(s_{i}, t_{i}\right)$ of $[0, \lambda)(i=1, \ldots, k, k \in \mathbb{N})$ satisfies

$$
\sum_{i=1}^{k}\left(t_{i}-s_{i}\right)<\delta, \text { then } \sum_{i=1}^{k}\left|F\left(t_{i}\right)-F\left(s_{i}\right)\right|<\varepsilon .
$$

Let $\varepsilon>0$ be arbitrary. By the assumption that $\lim _{t \rightarrow 0^{+}} F(t)=F(0)=0$, there exists $\delta_{1}=\delta_{1}(\varepsilon)>0$ such that $F(t) \leqslant F\left(\delta_{1}\right)<\varepsilon / 2$ for each $t \in[0, \delta]$. Moreover, by inequality (7), we have

$$
\frac{F(a)}{W(a)}=\frac{F(a)-F(0)}{W(a)-W(0)} \geqslant \frac{F\left(t_{1}\right)-F\left(t_{0}\right)}{W\left(t_{1}\right)-W\left(t_{0}\right)}
$$


for each $a \leqslant t_{0}<t_{1} \in I$. This means that

$$
0 \leqslant F\left(t_{1}\right)-F\left(t_{0}\right) \leqslant \frac{F(a)}{W(a)}\left(W\left(t_{1}\right)-W\left(t_{0}\right)\right),
$$

and since the function $W$ is absolutely continuous, there exists $\delta_{2}=\delta_{2}(\varepsilon)>0$ such that implication (10) is fulfilled for $\bar{\varepsilon}:=\frac{W(a) \varepsilon}{2 F(a)}$ and the function $W$ in the place of $F$. Taking $\delta=\min \left(\delta_{1}, \delta_{2}\right)$, we obtain implication (10) for $F$.

For a given increasing non-negative function $F$ we denote by $F^{b}$ its least $w$-concave majorant. The following theorem concerns the existence of such a majorant.

\subsection{Theorem.}

(i) (see [37, Thm 3.6.2] and [43, Thm 2.8]) Let $w \in \mathcal{W}$. If $F$ is an increasing non-negative function on I which has a $w$-concave majorant, then $F$ has a unique least $w$-concave majorant.

(ii) (see [37, page 69]) If we assume additionally that $\lim _{t \rightarrow 0^{+}} F(t)=F(0)=0$, then also $\lim _{t \rightarrow 0^{+}} F^{b}(t)=F^{b}(0)=0$.

Proof. (i). Define $F^{b}(t):=\inf \{G(t): G$ is aw-concave majorant of $F\}$. By the assumption, the infimum is taken over a nonempty set, so $F^{b}(t)<\infty$ for every $t \in I$. Evidently $F \leqslant F^{b}$, therefore, we only need to show that $F^{b}$ is $w$-concave.

Let $0 \leqslant a<b \in I$, and let $L(t)=c W(t)+d$ be such that $F^{b}(a)=L(a)$ and $F^{b}(a)=L(a)$. Then, for any $w$-concave majorant $G$ of $F$ and any $w$-affine function $L_{g}(t)=c_{g} W(t)+d_{g}$ such that $G(a)=L_{g}(a)$ and $G(b)=L_{g}(b)$, we have $L(a)=$ $F^{b}(a) \leqslant G(a)=L_{g}(a)$ and $L(b)=F^{b}(b) \leqslant G(b)=L_{g}(b)$. Hence, by the monotonicity of the function

$$
\left(L-L_{g}\right)(t)=\left(c-c_{g}\right) W(t)+\left(d-d_{g}\right),
$$

we get that $L(t) \leqslant L_{g}(t)$ for every $t \in(a, b)$ and, in consequence, $L(t) \leqslant G(t)$ for every $t \in(a, b)$. Finally, since $G$ was arbitrary, we obtain $L(t) \leqslant F^{b}(t)$ for every $t \in(a, b)$.

(ii). Suppose to the contrary that there exist $\varepsilon>0$ and a strictly decreasing sequence $\left(t_{n}\right)$ such that $\lim _{n \rightarrow \infty} t_{n}=0$ and $F^{b}\left(t_{n}\right) \geqslant \varepsilon$ for all $n \in \mathbb{N}$. Since $F^{b}$ is $w$-concave, we get that $F^{b}(t) \geqslant \varepsilon$ for every $t \in\left(0, t_{1}\right]$.

On the other hand, there exists $0<\delta<t_{1}$ such that $F(t) \leqslant \varepsilon / 2$ for $t \in[0, \delta]$. Defining

$$
F_{\varepsilon}(t)=\frac{F^{b}(\delta)-\frac{\varepsilon}{2}}{W(\delta)} W(t)+\frac{\varepsilon}{2}
$$

for $t \in I$, we have $F(t) \leqslant F_{\varepsilon}(t)$ for the same $t$ (more precisely, for $t>\delta$ we have $F(t) \leqslant$ $\left.F^{b}(t)<F_{\varepsilon}(t)\right)$. Moreover, since $F_{\varepsilon}$ is $w$-affine, $F_{\varepsilon}$ is a $w$-concave majorant of $F$. At the same time $F_{\varepsilon}(t)<F^{b}(t)$ for $t \in(0, \delta)$, which gives a contradiction with the definition of $F^{b}$. 
Note that if $F$ is a bounded function of the form $F(t)=\int_{0}^{t} f(s) d s$ (in particular, if supp $f \subset[a, b])$ or the function $\frac{f}{w}$ is bounded, then there exists a $w$-concave majorant of $F$ (a constant function is always $w$-affine) and, in consequence, there exists the least $w$-concave majorant $F^{b}$ (see [43]). In general, the situation is more delicate. The following examples illustrate situations that may happen. In the first example we show a function $F$ which does not have a $w$-concave majorant; in the second - we show that the boundedness of the function $\frac{f}{w}$ is not necessary for the existence of a $w$-concave majorant.

2.5. Example. For $t>0$, let $w(t)=\frac{1}{2} \cdot t^{-\frac{1}{2}}$ and $f(t)=\frac{3}{4} \cdot t^{-\frac{1}{4}}$. Then $W(t)=t^{\frac{1}{2}}$ and $F(t)=t^{\frac{3}{4}}$. We will show that a $w$-concave majorant of $F$ does not exist. Suppose to the contrary that there exists a $w$-concave function $G$ such that $F(t) \leqslant G(t)$ for all $t \in[0, \infty)$. By inequality (7), we have

$$
\frac{G(1)-G(0)}{W(1)-W(0)} \geqslant \frac{G(t)-G(0)}{W(t)-W(0)}
$$

for $t \geqslant 1$, whence

$$
G(t) \leqslant[G(1)-G(0)] \cdot W(t)+G(0)=[G(1)-G(0)] \cdot t^{\frac{1}{2}}+G(0) \leqslant G(1) \cdot t^{\frac{1}{2}}
$$

for $t \geqslant 1$. At the same time we have $G(1) \cdot t^{\frac{1}{2}}<t^{\frac{3}{4}}=F(t)$ for $t>G(1)^{4}$. Finally, $G(t)<F(t)$ for $t>G(1)^{4}$, which gives a contradiction.

2.6. Example. For $t>0$, let $f(t)=\min \left(1, t^{-2}\right)$ and $w(t)=\min \left(1, t^{-3}\right)$. Then $F(t)=$ $W(t)=t$ for $t \leqslant 1$, and $F(t)=2-t^{-1}$ and $W(t)=\frac{3}{2}-\frac{1}{2} t^{-2}$ for $t \geqslant 1$. It is easy to see that the function $G(t)=\frac{4}{3} W(t)$ is a $w$-concave majorant of $F$ (in Example 2.15 we will show that $\left.F^{b}(t)=\frac{4}{3} W(t)\right)$. Moreover, $\frac{f(t)}{w(t)}=\max (1, t)$, so the function $\frac{f}{w}$ is not bounded.

Finally we are in a position to define a level function (in the sense of Halperin).

2.7. Definition. Let $w \in \mathcal{W}$, and let $f$ be a non-negative and locally integrable function on $I$ such that the function $F(t)=\int_{0}^{t} f(s) d s$ has the least $w$-concave majorant $F^{b}$. Then $f^{o}$ such that

$$
F^{b}(t)=\int_{0}^{t} f^{o}(s) d s \quad \text { for } t \in I
$$

is called the level function of $f$.

Notice that for a function $F$ as above, by Theorem 2.3 the majorant $F^{b}$ is an absolutely continuous function, so the only requirement for the existence of $f^{o}$ is that $F^{b}$ exist.

At this point, it must be stressed that the above definition gives the level function in the sense of Halperin (its constructive definition will be presented below). A slightly 
different approach was proposed by Sinnamon. For a given positive function $g$ he defines a function $G_{w}$ as

$$
G_{w}(t)=\int_{0}^{t} g(s) w(s) d s \quad \text { for } t \in I,
$$

and then looks for a derivative of the least $w$-concave majorant $G^{b}$ of $G_{w}$. Now using part (iii) of Theorem 2.1, we see that $G^{b}$ may be written as

$$
G^{b}(t)=\int_{0}^{t} g^{s}(s) w(s) d s \quad \text { for } t \in I,
$$

where $g^{s}$ is a decreasing function.

2.8. Definition. Let $w, g, G_{w}$, and $G^{b}$ be as above. Then $g^{s}$ such that

$$
G^{b}(t)=\int_{0}^{t} g^{s}(s) w(s) d s \quad \text { for } t \in I,
$$

is called a level function of $g$ (in the sense of Sinnamon).

Notice that this point of view allows a generalization to arbitrary Borel measure in place of $w(s) d s$, and such a general situation was investigated by Sinnamon (see $[43,44$, 46]). Lorentz's approach was similar, and his function $D^{o}$ is equal to $g^{s}$ (see [37] and [47]).

Before we present constructive ways to obtain the two kinds of level functions we will compare the level functions of Sinnamon's and of Halperin's. Let $f$ be a locally integrable nonnegative function on $I$, and let $F(t)=\int_{0}^{t} f(s) d s$. To get the same function in Sinnamon's approach, we need to take $g=f / w$. Then $G_{w}=F$, where $G_{w}(t)=\int_{0}^{t} \frac{f(s)}{w(s)} w(s) d s$, and so their least $w$-concave majorants are the same, i.e.

$$
F^{b}(t)=\int_{0}^{t} f^{o}(s) d s=\int_{0}^{t}\left(\frac{f}{w}\right)^{s}(s) w(s) d s=G^{b}(t) \quad \text { for } t \in I .
$$

By differentiating both sides we obtain the required formula

$$
f^{o}=\left(\frac{f}{w}\right)^{s} w
$$

In particular, the above formula shows that $f^{o} / w$ is decreasing even if $f$ is not.

Now we proceed to present constructive ways of obtaining level functions. Halperin's point of view is based on condition (ii) of Theorem 2.1 (cf. [12]). The second method is due to Lorentz [37] and Sinnamon [43]; in this case, condition (iii) of the same theorem is in use.

Let $f$ be a non-negative and locally integrable function on $I$. For $0 \leqslant a<b \in I$, we define, after Halperin [12],

$$
W(a, b)=\int_{a}^{b} w(s) d s, \quad F(a, b)=\int_{a}^{b} f(s) d s, \quad R(a, b)=\frac{F(a, b)}{W(a, b)} ;
$$


we also define

$$
R(a, \infty)=\limsup _{t \rightarrow \infty} R(a, t) .
$$

2.9. Remark. When there exists a $w$-concave majorant $G$ of $F$ we have $R(a, \infty)<\infty$ for every $a \in[0, \infty)$. Indeed, by inequality (7), we have

$$
R_{G}(0,1):=\frac{G(0,1)}{W(0,1)}=\frac{G(1)-G(0)}{W(1)-W(0)} \geqslant \frac{G(t)-G(a)}{W(t)-W(a)}
$$

for every $a \geqslant 0$ and every $t \geqslant 1$. Hence,

$$
R(a, t)=\frac{F(t)-F(a)}{W(t)-W(a)} \leqslant \frac{G(t)-G(a)+G(a)-F(a)}{W(t)-W(a)} \leqslant R_{G}(0,1)+\frac{G(a)-F(a)}{W(2 a)-W(a)}
$$

for $a \geqslant 0$ and $t \geqslant \max (1,2 a)$. Whence we get $R(a, \infty)<\infty$ for every $a \in[0, \infty)$.

Let $f \geqslant 0$ be a locally integrable function on $I$. An interval $(a, b) \subset I$ is called a level interval of $f$ with respect to $w$ if $b<\infty$ and

$$
R(a, t) \leqslant R(a, b) \text { for all } t \in(a, b) .
$$

Analogously, the interval $(a, \infty)$ is called a degenerate level interval of $f$ with respect to $w$ if

$$
R(a, t) \leqslant R(a, \infty) \text { for all } t \in(a, \infty) .
$$

If a level interval is not contained in any larger level interval, then it is called a maximal level interval of $f$ with respect to $w$, or just a maximal level interval of $f$. We will use the abbreviations l.i. and m.l.i., respectively.

It is easy to see that $(a, \infty)$ is a level interval of $f$ if $f(t)=0$ for a.a. $t \in(a, \infty)$ and that $(a, \infty)$ is also a maximal level interval of $f$ if, additionally, $f$ is decreasing and $\operatorname{supp} f=[0, a]$. However, these rather trivial level intervals will often be omitted by us in further considerations.

We will need the following properties of level intervals.

2.10. Theorem ([12, Thm 3.1]). Let $0 \leqslant f$ on $I=[0, \lambda)$ be such that $R(I):=R(0, \lambda)<\infty$. The following assertions are true.

(i) If $\left(a_{1}, b_{1}\right)$ and $\left(a_{2}, b_{2}\right)$ are level intervals of $f$, where $a_{1}<a_{2}<b_{1}<b_{2}$, then $\left(a_{1}, b_{2}\right)$ is a level interval.

(ii) Every level interval is contained in a maximal level interval.

(iii) Maximal level intervals are pairwise disjoint and, as a consequence, there is at most a countable number of them. 
Proof. (i). Let $\left(a_{1}, b_{1}\right)$ and $\left(a_{2}, b_{2}\right)$ be level intervals of $f$. By the definition of $R(a, b)$ and by (6) we have

$$
R\left(a_{1}, a_{2}\right) \leqslant R\left(a_{1}, b_{1}\right) \leqslant R\left(a_{2}, b_{1}\right) \leqslant R\left(a_{2}, b_{2}\right) \leqslant R\left(b_{1}, b_{2}\right)
$$

and, in consequence,

$$
R\left(a_{1}, b_{1}\right) \leqslant R\left(a_{1}, b_{2}\right) \leqslant R\left(b_{1}, b_{2}\right) \text { and } R\left(a_{1}, a_{2}\right) \leqslant R\left(a_{1}, b_{2}\right) \leqslant R\left(a_{2}, b_{2}\right) .
$$

Hence, $R\left(a_{1}, t\right) \leqslant R\left(a_{1}, b_{1}\right) \leqslant R\left(a_{1}, b_{2}\right)$ for $t \in\left(a_{1}, b_{1}\right)$. Similarly, $R\left(a_{2}, t\right) \leqslant R\left(a_{2}, b_{2}\right) \leqslant$ $R\left(t, b_{2}\right)$ for $t \in\left(b_{1}, b_{2}\right)$; whence

$$
R\left(a_{1}, t\right) \leqslant R\left(a_{1}, b_{2}\right) \leqslant R\left(t, b_{2}\right),
$$

for $t \in\left(a_{1}, b_{2}\right)$. Thus $\left(a_{1}, b_{2}\right)$ is a level interval.

Assertions (ii) and (iii) follow directly from (i), which completes the proof.

Let us forget for a moment Definition 2.7 and define once again the level function $f^{o}$ of $0 \leqslant f$ as Halperin actually did it.

2.11. Definition. Let $w \in \mathcal{W}$. For $0 \leqslant f$ satisfying $R(I)<\infty$, let $\left\{\left(a_{n}, b_{n}\right)\right\}$ be a sequence of all maximal level intervals of $f$. Define

$$
f^{o}(t)= \begin{cases}R\left(a_{n}, b_{n}\right) w(t) & \text { for } t \in\left(a_{n}, b_{n}\right), n \in \mathbb{N}, \\ f(t) & \text { otherwise. }\end{cases}
$$

We need to show that Halperin's definition gives the same $f^{o}$ as Definition 2.7. Let us first notice the following fact.

2.12. Lemma. Let $f$ and $f^{o}$ be as in Definition 2.11. If $(r, s)$ is a level interval of $f^{o}$, then $f^{o}(t)=k w(t)$ for all $t \in(r, s)$ and some constant $k \geqslant 0$.

Proof. It will cause no confusion if we put $F^{o}(a, b)=\int_{a}^{b} f^{o}(s) d s$ and $R^{o}(a, b)=\frac{F^{o}(a, b)}{W(a, b)}$. First of all, notice that if $(a, b)$ is a l.i. of $f$, then it is also a li.i. of $f^{o}$, since $f^{o}(t)=k w(t)$ for all $t \in(a, b)$ and a constant $k$, which means that $R^{o}(a, t)=R^{o}(a, b)$ for all $t \in(a, b)$. Next, we claim that if $(a, b)$ is a m.l.i. of $f^{o}$, then neither $a$ nor $b$ is in a level interval of $f$. Indeed, if $a \in(c, d)$, and $(c, d)$ is a li. of $f$, then from the preceding considerations it follows that $(c, d)$ is a l.i. of $f^{0}$, which, in light of Theorem $2.10(\mathrm{i})$, contradicts the maximality of $(a, b)$ for $f^{o}$. Similarly for $b$. Therefore, by the definition of $f^{o}$, for $t \in(a, b)$ we have

$$
R(a, t) \leqslant R^{o}(a, t) \leqslant R^{o}(a, b)=R(a, b) .
$$

As a result, if $(a, b)$ is a m.l.i. of $f^{o}$ containing $(r, s)$, then $(a, b)$ is also a m.l.i. of $f$ and $f^{o}(t)=R(a, b) w(t)$ for $t \in(a, b)$, and the lemma follows. 
2.13. Theorem. Let $f$ and $w$ satisfy the conditions of Definition 2.11. Then for $f^{o}$ defined in Definition 2.11, the function

$$
F^{b}(t)=\int_{0}^{t} f^{o}(s) d s
$$

is the least $w$-concave majorant of $F$.

Proof. Once we prove that the function $F^{b}$ is $w$-concave, the rest of the assertion will be clear. We want to use part (ii) of Theorem 2.1, so we need to check that for arbitrary $0 \leqslant a<t<b<\infty$,

$$
R^{o}(a, t) \geqslant R^{o}(a, b) .
$$

Suppose, to the contrary, that this is not the case, that is, that $R^{o}\left(a, t_{0}\right)<R^{o}(a, b)$ for some $0 \leqslant a<t_{0}<b<\infty$. Then either, by the continuity of $R^{o}(a, \cdot)$, there is $d<b$ such that $R^{o}(a, d)=R^{o}(a, b)$, and $R^{o}(a, t) \leqslant R^{o}(a, b)$ for $t_{0}<t<d$, or $R^{o}(a, t) \leqslant R^{o}(a, b)$ for $t_{0}<t<b$, and we put $d=b$ in the latter case. We then have $R^{o}(a, t) \leqslant R^{o}(a, d)$ for $t \in\left[t_{0}, d\right)$. On the other hand, we know that $R^{o}(a, t) \leqslant R^{o}(a, d)$ if and only if $R^{o}(t, d) \geqslant$ $R^{o}(a, d)$ (see (6)). In particular, $R^{o}\left(t_{0}, d\right)>R^{o}(a, d)=R^{o}(a, b)$. As before, either there is $a<c$ such that $R^{o}(c, d)=R^{o}(a, d)$, and $R^{o}(c, t) \geqslant R^{o}(c, d)$ for $c<t<t_{0}$, or $R^{o}(a, t) \geqslant$ $R^{o}(a, d)$ for $c<t<t_{0}$, and we put $c=a$ in this case. Using once again the respective equivalence, we conclude that $R^{o}(c, t) \leqslant R^{o}(c, d)$ for all $t \in(c, d)$. This means that $(c, d)$ is a level interval of $f^{o}$ with respect of $w$, and so $f^{o}(t)=k w(t)$ for all $t \in(c, d)$ and some constant $k>0$. But then

$$
k=R^{o}(c, d)=R^{o}\left(c, t_{0}\right),
$$

and we will see that this leads to a contradiction. Indeed, we assumed that $R^{o}\left(a, t_{0}\right)<$ $R^{o}(a, b)=R^{o}(a, d)$, which gives $R^{o}\left(t_{0}, d\right)>R^{o}(a, d)=R^{o}(c, d)$. Using (6) we obtain $R^{o}\left(c, t_{0}\right)<R^{o}(c, d)$, and the contradiction is shown.

2.14. Corollary. If $0 \leqslant f$ satisfies the condition $R(0, \infty)<\infty$, then $F$ has the least $w$-concave majorant.

Before closing this section, let us comment a little on Halperin's construction of the level functions. Condition (ii) in Theorem 2.1 and the definition of level intervals tell us that level intervals are, roughly speaking, the intervals on which $F$ loses its $w$-concavity and needs to be replaced by a $w$-affine function. On the other hand, starting with the least majorant $F^{b}$ of $F$, we may look for intervals (they are intervals because both $F^{b}$ and $F$ are continuous) on which $F^{b}(t)>F(t)$. It is, however, important to understand that even if $F^{b}(t)>F(t)$ on an interval $(a, b)$, and $F^{b}(a)=F(a), F^{b}(b)=F(b)$, the interval $(a, b)$ is a level interval of $f$ but need not be a maximal level interval (see example below). 
Maximal level intervals are the ones on which $F^{b}$ is $w$-affine is not $w$-affine on any larger interval.

\subsection{Example.}

(i) For $f=c \cdot w, c>0$, we see that $(0, \infty)$ is a maximal level interval, $R(0, \infty)=c, f^{o}=f$, hence $F^{b}=F$.

(ii) For the functions $w$ and $f$ defined in Example 2.6, the interval $(0, \infty)$ is a maximal level interval, $R(0, \infty)=\frac{4}{3}, F^{b}(t)=\frac{4}{3} W(t)>F(t)$ for any $t \in(0, \infty)$, and $\lim _{t \rightarrow \infty} F^{b}(t)=\lim _{t \rightarrow \infty} F(t)=2$.

(iii) Now let $w(t)=1$ for $t \in[0,1), w(t)=\frac{1}{2 t}$ for $t \geqslant 1$, and $f(t)=\min \left(1, \frac{1}{t}\right)$ for $t \in[0, \infty)$. Then, as above, $(0, \infty)$ is a maximal level interval, $R(0, \infty)=2, F^{b}(t)=2 W(t)>$ $F(t)$ for any $t \in(0, \infty)$, but $\lim _{t \rightarrow \infty} F^{b}(t)=\lim _{t \rightarrow \infty} F(t)=\infty$.

(iv) Let $I=[0,11], w(t)=11-t$, and

$$
f(t)= \begin{cases}11-2 t & \text { for } t \in[0,1], \\ 0.9(11-t) & \text { for } t \in[1,3], \\ 1.44(11-2 t) & \text { for } t \in[3,4] \\ 4.32 & \text { for } t \in\left[4, t_{1}\right], \text { where } 4<t_{1} \leqslant 10 \\ 1.5 \frac{11-1.1 \cdot t}{11-1.1 \cdot t_{1}} & \text { for } t \in\left(t_{1}, 10\right], \\ 0 & \text { for } t \in[10,11] .\end{cases}
$$

First, note that, given any any $a \in[0,1)$, the function $R(a, t)=\frac{11-(a+t)}{11-(a+t) / 2}$ is strictly decreasing for $t \in(a, 1]$ and

$$
1>\frac{11-2 a}{11-a}>R(a, t)>\frac{10-a}{10.5-a / 2}>\frac{9}{10}
$$

for the same $a$ and $t$. In turn, the function $R(1, t)$ is equal to 0.9 on the interval $(1,3]$, strictly decreasing on $\left(3, \min \left(t_{0}, t_{1}\right)\right)$, and strictly increasing on $\left(t_{0}, t_{1}\right)$, where $t_{0}=(-4.68+6 \sqrt{23.85}) / 4.32$ and $t_{0}<t_{1}$. In particular, if $t_{1}=\frac{31+4 \sqrt{11}}{5}$, we have $R\left(1, t_{1}\right)=0.9$, and there is exactly one maximal level interval $\left(1, t_{1}\right)$ (see Figures 1 and 2). Finally, it is worth noticing that if $4<t_{1}<\frac{31+4 \sqrt{11}}{5}$, then there exist two maximal level intervals $(1,3)$ and $\left(t_{2}, t_{1}\right)$, where $t_{2} \in(3,4)$ (for example, for $t_{1}=7$ we get $\left.t_{2}=(68-3 \sqrt{97}) / 11 \approx 3.4958\right)$.

(v) Let $w(t)=\frac{1}{2 \sqrt{t}}$, and define $f$ as follows

$$
f(t)= \begin{cases}w(t) & \text { for } t \in[0,1) \\ 1 / 3 & \text { for } t \in[1,4) \\ 0 & \text { for } t \in[4, \infty)\end{cases}
$$




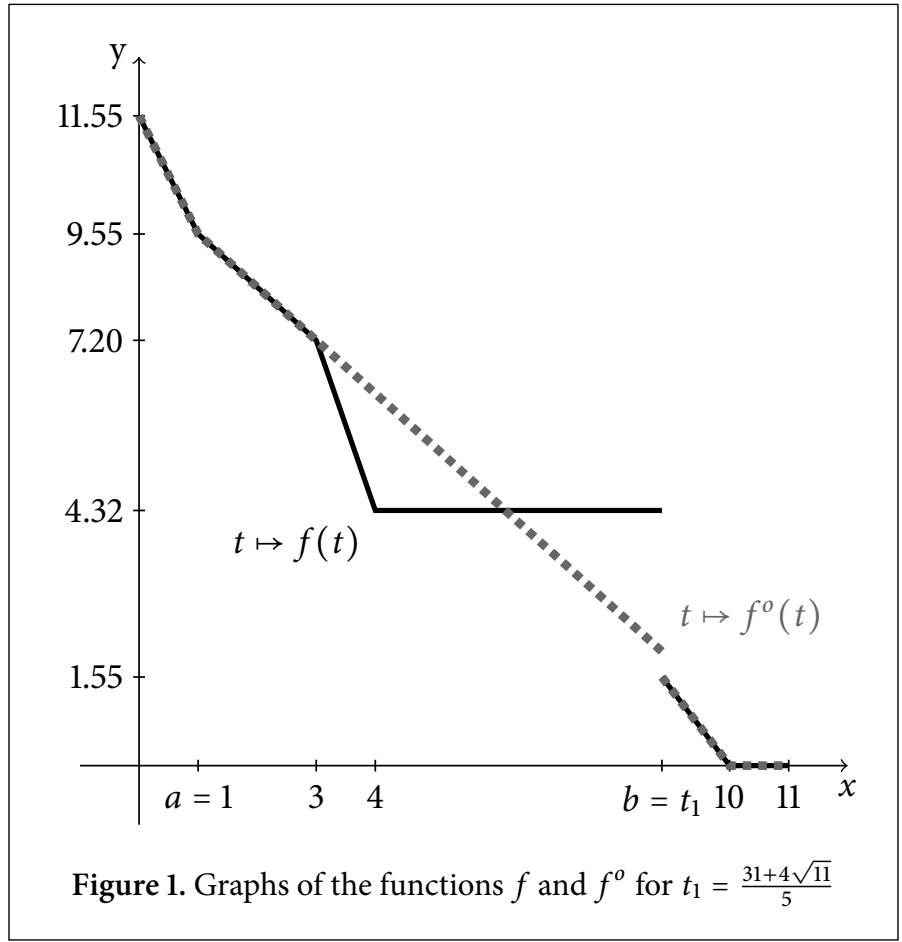

It is easy to see that $R(0, t)=R(0,4)=1$ for $t \in(0,1]$, and $R(0, t)<R(0,4)$ for $t \in(1,4)$. Therefore, $(0,4)$ is a m.l.i. of $f$ and $f^{o}=w \chi_{[0,4)}$. If we took $f(t)=a<1 / 3$ on $[1,4)$ in the above definition, then we would have two maximal level intervals: $(0,1)$ and $(1,4)$. Moreover, taking $f(t)=\frac{1}{4 t^{3 / 4}}$ on $[0,1)$, we have a m.l.i. $(1,4)$ while $F$ is $w$-concave on $[0,1)$, which is easiest to see by writing

$$
F(t)=t^{1 / 4}=\int_{0}^{t} g(s) \frac{1}{2 \sqrt{s}} d s \quad \text { for } t \in(0,1)
$$

where $g(s)=\frac{1}{2 t^{1 / 4}}$ is a decreasing function. It may also be instructive to consider

$$
f_{b}(t)= \begin{cases}w(t) & \text { for } t \in[0,1), \\ b w(t)+(1-b) / 3 & \text { for } t \in[1,4), \\ 0 & \text { for } t \in[4, \infty)\end{cases}
$$

with $b \in(0,1)$. For all such $b$ we have still $f_{b}^{o}=w \chi_{[0,4)}$. 


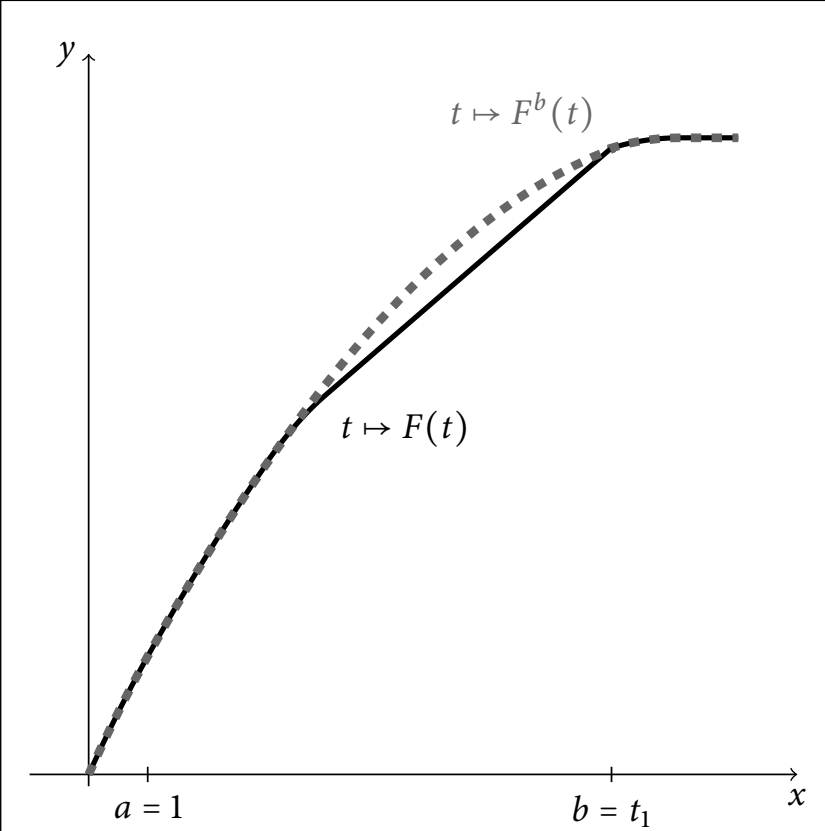

Figure 2. Graphs of the functions $F$ and $F^{b}$ for $t_{1}=\frac{31+4 \sqrt{11}}{5}$

As a final note, applying the above considerations to Sinnamon's level function $g^{s}$, we see that

$$
g^{s}(t)= \begin{cases}\frac{\int_{a_{n}}^{b_{n}} g(s) w(s) d s}{\int_{a_{n}}^{b_{n}} w(s) d s} & \text { for } t \in\left(a_{n}, b_{n}\right), n \in \mathbb{N}, \\ g(t) & \text { otherwise, }\end{cases}
$$

where $\left\{\left(a_{n}, b_{n}\right)\right\}$ is the sequence of all maximal level intervals of $g w$ with respect to $w$; in the case of the degenerate l.i. we are to understand

$$
\frac{\int_{a_{n}}^{\infty} g(s) w(s) d s}{\int_{a_{n}}^{\infty} w(s) d s}
$$

as

$$
\limsup _{b \rightarrow \infty} \frac{\int_{a_{n}}^{b} g(s) w(s) d s}{\int_{a_{n}}^{b} w(s) d s}
$$


In another words, here maximal level intervals are the maximal intervals on which $g^{s}$ is constant. It also makes sense to write $g^{s}$ in terms of an averaging operator with respect to the measure $d \mu=w(t) d t$; namely, when all m.l.i.'s are proper, then

$$
g^{s}=A g
$$

where $A$ is defined by

$$
A g=\sum_{n}\left(\frac{1}{\mu\left(\left(a_{n}, b_{n}\right)\right)} \int_{\left(a_{n}, b_{n}\right)} g(s) d \mu\right) \chi_{\left(a_{n}, b_{n}\right)}+g \chi_{I \cup_{n}\left(a_{n}, b_{n}\right)}
$$

(cf. $[44,45]$ and $[46]$ ).

Notice also that although Halperin's definition shows how to construct level functions, we do not have a way of determining level intervals. This gap may be filled by an algorithm presented in [19], which shows how to build the level function of a simple function, or, more precisely, how to determine the level intervals of a simple function (in fact, the algorithm produces yet another kind of a level function but it may readily be translated to a Halperin's level function).

\section{Sawyer's formula, Sinnamon's down spaces and level functions, and Cesàro spaces}

In this section we fix $I=[0, \infty)$ and the weight function $w \in \mathcal{W}$ with $W(\infty)=\infty$. For the weighted space $L_{p}(w)$, given by the norm $\|f\|_{L_{p}(w)}=\left(\int_{0}^{\infty}|f(t)|^{p} w(t) d t\right)^{1 / p}$ with $1<p<\infty$ and a positive weight $w$, the Köthe dual space $\left(L_{p}(w)\right)^{\prime}$ is known, from classical Hölder-Rogers inequality with equality for some particular functions, to be $L_{q}(w)$, where $1 / p+1 / q=1$, with equality of norms. In particular,

$$
\|f\|_{\left(L_{p}(w)\right)^{\prime}}=\sup \left\{\int_{0}^{\infty} \frac{|f(t)|}{w(t)} g(t) w(t) d t: 0 \leqslant g,\|g\|_{L_{p}(w)} \leqslant 1\right\}=\left\|\frac{f}{w}\right\|_{L_{q}(w)} .
$$

In 1953, Halperin considered the following functional

$$
S\left(f, L_{p}(w)\right):=\sup \left\{\int_{0}^{\infty}|f(t)| g(t) d t: 0 \leqslant g \downarrow,\|g\|_{L_{p}(w)} \leqslant 1\right\},
$$

which may be regarded as a kind of Hölder-Rogers inequality (compared with the preceding formula, we just restrict to decreasing functions), and he called it the $D$-type Hölder's inequality. Halperin determined the direct formula for this functional:

$$
S\left(f, L_{p}(w)\right)=\left(\int_{I}\left(\frac{f^{o}(t)}{w(t)}\right)^{q} w(t) d t\right)^{1 / q},
$$

where $1 / p+1 / q=1$ and $f^{o}$ is the level function of $f$ with respect to $w$. 
In 1990, Sawyer [41] mentions Halperin's result on page 146, but instead of equality (1) he found the following equivalence formula for $S\left(f, L_{p}(w)\right)$

$$
S\left(f, L_{p}(w)\right) \approx\left(\int_{0}^{\infty}\left(\frac{\int_{0}^{x}|f(t)| d t}{W(x)}\right)^{q} w(x) d x\right)^{1 / q},
$$

(see also [28, Thm 6.3]; simpler proofs of Sawyer's equivalence formula were found by Stepanov [48] and Carro-Soria [4, Thm 3.1]). If we put

$$
S_{w} f(x)=\frac{1}{W(x)} \int_{0}^{x} f(t) d t, \text { then } S\left(f, L_{p}(w)\right) \approx\left\|S_{w}|f|\right\|_{L_{q}(w)} .
$$

Of course, if $w \equiv 1$ and $1<p<\infty$, we get

$$
S\left(f, L_{p}\right) \approx\|C|f|\|_{L_{q}}, \quad \text { where } C f(x)=S_{1} f(x)=\frac{1}{x} \int_{0}^{x} f(t) d t
$$

is the usual Cesàro operator.

For $0<p \leqslant 1$, a Sawyer-type duality formula was proved by Carro-Soria [5, Thm 3.2] in the equivalence form and by Heinig-Maligranda [14] in the equality form (see also [28, Thm 6.5]): $S\left(f, L_{p}(w)\right)=\sup _{x>0} W(x)^{-1 / p} \int_{0}^{x}|f(t)| d t$.

In 1996, Heinig-Kufner [13, Thm 2.2] proved a duality theorem in weighted Orlicz function spaces (and in weighted Orlicz sequence spaces): If the $\mathrm{N}$-function $\varphi$ and its complementary function $\psi$ satisfy the $\Delta_{2}$-condition, that is, if there are constants $C, D>0$ such that $\varphi(2 u) \leqslant C \varphi(u)$ and $\psi(2 u) \leqslant C \psi(u)$ for all $u>0$, then

$$
S\left(f, L_{\varphi}(w)\right) \approx\left\|\frac{\int_{0}^{x}|f(t)| d t}{W(x)}\right\|_{L_{\psi}(w)},
$$

where the weighted Orlicz space $L_{\varphi}(w)$ consists of all $f \in L^{0}$ for which the Luxemburg-Nakano norm $\|f\|_{L_{\varphi}(w)}=\inf \left\{\lambda>0: \int_{0}^{\infty} \varphi\left(\frac{|f(t)|}{\lambda}\right) w(t) d t \leqslant 1\right\}$ is finite.

Sawyer's formula was then generalized to symmetric spaces over general measure spaces. To formulate duality results for $w$-symmetric spaces $X(w)$, we need the linear operator of Cesàro (or Hardy)-type $C_{w}$ and its formal adjoint $C_{w}^{\prime}$, defined by

$$
C_{w} f(x)=\frac{1}{W(x)} \int_{0}^{x} f(t) w(t) d t \quad \text { and } \quad C_{w}^{\prime}(x)=\int_{x}^{\infty} \frac{f(t)}{W(t)} w(t) d t .
$$

By a change of variables we obtain equalities

$$
\left(C_{w} f\right)\left(W^{-1}(x)\right)=\frac{1}{x} \int_{0}^{W^{-1}(x)} f(t) w(t) d t=\frac{1}{x} \int_{0}^{x} f\left(W^{-1}(s)\right) d s=C\left(f \circ W^{-1}\right)(x)
$$

and

$$
\left(C_{w}^{\prime} f\right)\left(W^{-1}(x)\right)=\int_{W^{-1}(x)}^{\infty} \frac{f(t)}{W(t)} w(t) d t=\int_{x}^{\infty} \frac{f\left(W^{-1}(s)\right)}{s} d s=C^{\prime}\left(f \circ W^{-1}\right)(x) .
$$


Thus, if the operator $C$ is bounded on $X$ (where $X$ stands for the canonical representation of $X(w))$, then the operator $C_{w}$ is bounded on $X(w)$ and $\left\|C_{w}\right\|_{X(w) \rightarrow X(w)} \leqslant\|C\|_{X \rightarrow X}$. Indeed, by the above equality and (2)

$$
\left\|C_{w} f\right\|_{X(w)}=\left\|C\left(f \circ W^{-1}\right)\right\|_{X} \leqslant\|C\|_{X \rightarrow X}\left\|f\left(W^{-1}\right)\right\|_{X}=\|C\|_{X \rightarrow X}\|f\|_{X(w)} .
$$

Similarly, if $C^{\prime}$ is bounded on $X$, then $C_{w}^{\prime}$ is bounded on $X(w)$ and $\left\|C_{w}^{\prime}\right\|_{X(w) \rightarrow X(w)} \leqslant$ $\left\|C^{\prime}\right\|_{X \rightarrow X}$.

For $w$-symmetric spaces $X(w)$, Gogatishvili and Pick [10] considered Sawyer's functional of the form

$$
S(f, X(w)):=\sup \left\{\int_{0}^{\infty}|f(t)| g(t) d t: 0 \leqslant g \downarrow,\|g\|_{X(w)} \leqslant 1\right\} .
$$

They proved [10, Thm 4.1] that if the operators $C, C^{\prime}$ are bounded on the symmetric space $X$ with the Fatou property (where $X$ is the canonical representation of $X(w)$ as in the introduction), then

$$
S(f, X(w)) \approx\left\|\int_{W^{-1}(x)}^{\infty} \frac{|f(t)|}{W(t)} d t\right\|_{X^{\prime}} \approx\left\|\left(S_{w}|f|\right)\left(W^{-1}\right)\right\|_{X^{\prime}}=\left\|S_{w}|f|\right\|_{X(w)^{\prime}} .
$$

Of course, it is well known that $C, C^{\prime}$ are bounded on the symmetric space $X$ if and only if the Boyd indices are non-trivial (cf. [1, Thm 5.15; 3, Thm 3.4; 26, Chap. II, Thms 6.6 and 6.8] and definitions therein; see also [27]).

Kamińska and Mastyło [21] generalized Sawyer's formula to Banach function spaces $X(w)$ (over the measure space $(I, \mu)$ ), and even quasi-Banach function spaces. In $[21, \mathrm{Thm}$ 1.1] they proved the following result.

3.1. Theorem (Kamińska-Mastyło [21]). If the operators $C_{w}, C_{w}^{\prime}$ are bounded on the quasi-Banach function space $X(w)$, then

$$
S(f, X(w)):=\sup \left\{\int_{0}^{\infty}|f(t)| g(t) d t: 0 \leqslant g \downarrow,\|g\|_{X(w)} \leqslant 1\right\} \approx\left\|S_{w}|f|\right\|_{X(w)^{\prime}} .
$$

More precisely,

$$
\frac{1}{\left\|C_{w}^{\prime}\right\|_{X(w) \rightarrow X(w)}}\left\|S_{w}|f|\right\|_{X(w)^{\prime}} \leqslant S(f, X(w)) \leqslant\left\|C_{w}\right\|_{X(w) \rightarrow X(w)}\left\|S_{w}|f|\right\|_{X(w)^{\prime}}
$$

In particular, if $w \equiv 1$ and $C, C^{\prime}$ are bounded operators on a Banach function space $X$ on $[0, \infty)$, then

$$
S(f, X) \approx\left\|C^{\prime}|f|\right\|_{X^{\prime}}=\|C|f|\|_{X} .
$$

On the other hand, in 2001 Sinnamon introduced a down space $D(X(w))$ of a $w$ -symmetric space $X(w)$ (cf. [44], see also [42] and [43] where he considered an earlier 
version of the down spaces for $L_{p}(w)$ spaces, cf. [46, pp. 215-217]). Such a space is defined by the norm

$$
\|f\|_{D(X(w))}=\sup \left\{\int_{0}^{\infty}|f(t)| g(t) w(t) d t: 0 \leqslant g \downarrow,\|g\|_{X(w)^{\prime}} \leqslant 1\right\} .
$$

In fact, he described the down space $D(X(v))$ for a general $v$-symmetric space $X$ on $(-\infty, \infty)$ with $v$ the Borel measure, not necessarily absolutely continuous with respect to Lebesgue measure.

His description comes with an exact formula for a down norm in terms of level functions $f^{s}$ ([44, Corollary 2.4]) as well as an equivalent formula in terms of the operators $C_{w}$ and $C_{w}^{\prime}$ (cf. [44, Thm 3.1] and [46, Thms 2.2-2.4]).

3.2. Theorem (Sinnamon [44]). Let $X(w)$ be a w-symmetric space on $[0, \infty)$ with the Fatou property.

(i) If $f \in D(X(w))$, then $f^{s}$ is finite $\mu$-almost everywhere, belongs to $X(w)$, and

$$
\|f\|_{D(X(w))}=\left\|f^{s}\right\|_{X(w)},
$$

where $f^{s}$ stands for the level function of $f$ with respect to $w$ in the sense of Sinnamon.

(ii) We have $\|f\|_{D(X(w))} \approx\left\|C_{w}|f|\right\|_{X(w)}$ if and only if $C_{w}$ is bounded on $X(w)$.

(iii) If $C_{w}^{\prime}$ is a bounded operator on $X(w)$, then

$$
\begin{aligned}
\|f\|_{D(X(w))} & \leqslant\left\|C_{w}^{\prime}|f|\right\|_{X(w)} \leqslant\left\|C_{w}^{\prime}\right\|_{X(w) \rightarrow X(w)}\|f\|_{D(X(w))} \\
& =\left\|C_{w}\right\|_{X(w)^{\prime} \rightarrow X(w)^{\prime}}\|f\|_{D(X(w))} .
\end{aligned}
$$

The map $f \rightarrow f^{s}$ is not linear (even not sublinear), therefore equality (11) is a little surprising. In the proof of the above theorem Sinnamon used the following lemma, which is of independent interest (see [46, p. 211-213] for a nice explanation). Let $\mathbb{A}_{w}$ be the set of all averaging operators, i.e. the operators on $L^{1}$ over $d \mu=w(t) d t$ defined by

$$
A g(t)=\sum_{k}\left(\frac{1}{\int_{I_{k}} w(t) d t} \int_{I_{k}} g(s) w(t) d t\right) \chi_{I_{k}}(t)+g(t){\chi \mathbb{R}_{+} \backslash \cup_{k} I_{k}}_{(t),}
$$

where $\left(I_{k}\right)$ is a (possibly infinite) collection of pairwise disjoint bounded intervals.

3.3. Lemma (Sinnamon [46]). Let $0 \leqslant g \downarrow$ and $0 \leqslant f \in L^{0}(I)$. Then

$$
\sup _{0 \leqslant h \downarrow, h<w g} \int_{I} f(t) h(t) w(t) d t=\int_{I} f^{s}(t) g(t) w(t) d t=\sup _{A \in \mathbb{A}_{w}} \int_{I} f(t) A g(t) w(t) d t .
$$


From these considerations we conclude that the two kinds of Sawyer's formula, i.e. Kamińska-Mastyło's and Sinnamon's formulas are, roughly speaking, the same. In fact, for $0 \leqslant f$ we have $C_{w}(f)=S_{w}(f w)$, and the Fatou property gives $X(w)^{\prime \prime}=X(w)$, whence

$$
\|f\|_{D(X(w))}=S\left(f w, X(w)^{\prime}\right) \approx\left\|S_{w}(f w)\right\|_{X(w)}=\left\|C_{w} f\right\|_{X(w)} .
$$

In [46, Thm 2.1], Sinnamon proved a duality formula for the down space $D(X(w))$ if $X(w)$ is a Banach function space (see also [24, Thm D]). In a sense, the duality comes directly from the definition of the down norm, since it is defined by a duality formula.

3.4. Theorem (Sinnamon [46]). If $X(w)$ is a Banach function space on $[0, \infty)$, then the following duality formula holds

$$
\|f\|_{D(X(w))^{\prime}}=\sup \left\{\int_{0}^{\infty}|f(t) g(t)| w(t) d t:\|g\|_{D(X(w))} \leqslant 1\right\}=\|\widetilde{f}\|_{X(w)^{\prime}},
$$

where $\widetilde{f}$ is the decreasing majorant of $|f|$, that is, $\widetilde{f}(x)=\operatorname{ess} \sup _{t \geqslant x}|f(t)|, x \geqslant 0$.

Proof. For a $\mu$-measurable function $f$ we have

$$
\int_{I}|f(x) g(x)| w(x) d x \leqslant \int_{I} \widetilde{f}(x)|g(x)| w(x) d x \leqslant\|\widetilde{f}\|_{X(w)^{\prime}}\|g\|_{D(X(w))}
$$

and taking the supremum over all $\|g\|_{D(X(w))} \leqslant 1$ we obtain $\|f\|_{D(X(w))^{\prime}} \leqslant\|\widetilde{f}\|_{X(w)^{\prime}}$. On the other hand,

$$
\begin{aligned}
\int_{I} \widetilde{f}(x)|g(x)| w(x) d x & =\sup _{h<_{w} g} \int_{I} f(t) h(t) w(t) d t \leqslant \sup _{h<_{w} g}\|h\|_{D(X(w))}\|f\|_{D\left(X(w)^{\prime}\right)} \\
& \leqslant\|g\|_{D(X(w))}\|f\|_{D\left(X(w)^{\prime}\right)} \leqslant\|g\|_{X_{w}}\|f\|_{D\left(X(w)^{\prime}\right)},
\end{aligned}
$$

and taking the supremum over all $\|g\|_{X(w)} \leqslant 1$, we obtain $\|\widetilde{f}\|_{X(w)^{\prime}} \leqslant\|f\|_{D(X(w))^{\prime}}$.

From Theorems 3.1 and 3.4 we get that if $X$ is a Banach function space over the interval $([0, \infty),|\cdot|)$ with the Fatou property and such that $C, C^{\prime}$ are bounded on $X$, then

$$
\|f\|_{D(X)} \approx\|C|f|\|_{X} \approx\left\|C^{\prime}|f|\right\|_{X} \text { and }\|f\|_{D(X)^{\prime}} \approx\|\widetilde{f}\|_{X^{\prime}}
$$

The above result may also be regarded as a dual representation for Cesàro spaces, and vice versa: - duality in Cesàro spaces gives Sawyer-Sinnamon's formula, and hence we come back to the results of [30].

Indeed, if $C$ is bounded on $X$, then for the Cesàro space $C X=\left\{f \in L^{0}: C|f| \in X\right\}$ with norm $\|f\|_{C X}=\|C|f|\|_{X}$ we have $C X=D(X)$. Note also that $C L_{1}=\{0\}$ and $D\left(L_{1}\right)=L_{1}$.

Consequently, since $\widetilde{X}^{\prime}=(D(X))^{\prime}$ it follows that $\widetilde{X}^{\prime}=(C X)^{\prime}$. It seems to be of interest here to mention the assumptions that are needed in the three (most general) approaches under discussion. 
(a) In [21], $C_{w}, C_{w}^{\prime}$ have to be bounded on $X$, the measure $\mu$ is arbitrary, and the space $X$ need not be symmetric.

(b) In [30], only $C$ and the respective dilation operator $\sigma_{\tau}$ for $0<\tau<1$ have to be bounded on $X$, no symmetry of $X$ is required, and only Lebesgue measure is considered.

(c) Sinnamon's approach requires the boundedness of $C_{w}$ and $C_{w}^{\prime}$ on $X$, and $X$ has to be symmetric with respect to the measure $\mu$.

It is worth emphasizing that we have omitted the case of a finite measure in this section, whereas in Sawyer's formula (cf. [21]) appear some additional parts to cover this case as well. Additionally, for finite measures the down spaces are no longer equal to Cesàro spaces and the duality for the latter is also different (cf. [30-32]).

\section{Halperin spaces}

For $1 \leqslant q<\infty$, and a weight function $w \in \mathcal{W}_{d}$, the Halperin space $M_{q, w}$ is a subspace of $L^{0}(I,|\cdot|)$ defined by the norm $\|f\|_{M_{q, w}}=\left[f^{*}\right]_{q, w}$ where

$$
[f]_{q, w}=\left(\int_{I}\left(\frac{f^{o}(t)}{w(t)}\right)^{q} w(t) d t\right)^{1 / q}
$$

(we keep to the original notation of Halperin's [12]). This space is a (metric) Köthe dual of the Lorentz space $\Lambda_{p, w}$ given by the norm

$$
\|f\|_{\Lambda_{p, w}}=\left(\int_{I} f^{*}(t)^{p} w(t) d t\right)^{1 / p},
$$

where $1 / p+1 / q=1$. Following Halperin's and Lorentz's ideas, we will present a simple proof of this duality.

4.1. Theorem (Halperin, Lorentz). For a positive $f$ with $[f]_{q, w}<\infty$,

$$
[f]_{q, w}=\sup _{\substack{0 \leqslant g \downarrow \\\|g\|_{L_{p}(w)} \leqslant 1}} \int_{I} f(t) g(t) d t .
$$

In particular, if $f$ is decreasing, then

$$
[f]_{q, w}=\|f\|_{\left(\Lambda_{p, w}\right)^{\prime}}
$$

Proof. Notice that equality $[f]_{q, w}=\|f\|_{\left(\Lambda_{p, w}\right)^{\prime}}$ is obvious once we prove (12), since for a decreasing $f \in M_{q, w}$ it is enough to take decreasing $g$ 's in the formula

$$
\|f\|_{\left(\Lambda_{p, w}\right)^{\prime}}=\sup _{\substack{0 \leqslant g \\\|g\|_{\Lambda_{p, w}} \leqslant 1}} \int_{I} f(t) g(t) d t,
$$

and, moreover, we have $\|f\|_{\Lambda_{p, w}}=\|f\|_{L_{p}(w)}$ if $f=f^{*}$. 
Now we prove (12). Let $0 \leqslant f$ with $[f]_{q, w}<\infty$. First of all notice that by the definition of the level function $f^{o}$ of $f$ with respect to $w$, we have

$$
\int_{0}^{t} f(s) d s \leqslant \int_{0}^{t} f^{o}(s) d s \quad \text { for all } t \in I .
$$

Indeed, if $t$ does not belong to a level interval, then $\int_{0}^{t} f(s) d s=\int_{0}^{t} f^{o}(s) d s$, while for $t \in(a, b)$, where $(a, b)$ is a certain maximal level interval (which may be unbounded), we have

$$
\int_{a}^{t} f(s) d s \leqslant R(a, b) \int_{a}^{t} w(s) d s=\int_{a}^{t} f^{o}(s) d s .
$$

By Hardy's lemma, used for $0 \leqslant g \downarrow$ (cf. [12, Thm 4.1; 26, p. 72; 37, Thm 3.4.3]), and by Hölder-Rogers inequality, we obtain

$$
\begin{aligned}
\int_{I} f(s) g(s) d s & \leqslant \int_{I} f^{o}(s) g(s) d s=\int_{I} g(s) w^{1 / p}(s) f^{o}(s) w^{-1 / p}(s) d s \\
& \leqslant\left(\int_{I} g^{p}(s) w(s) d s\right)^{1 / p}\left(\int_{I}\left(f^{o}(s)\right)^{q} w^{-q / p}(s) d s\right)^{1 / q}=\|g\|_{L_{p}(w)}[f]_{q, w} .
\end{aligned}
$$

On the other hand, let $0 \leqslant f$ with $0<[f]_{q, w}<\infty$. Taking

$$
g_{1}=C\left(\frac{f^{o}}{w}\right)^{q-1} \quad \text { with } C=[f]_{q, w}^{-q / p}
$$

we obtain

$$
\left\|g_{1}\right\|_{L_{p}(w)}=C\left(\int_{I}\left(\frac{f^{o}(t)}{w(t)}\right)^{p(q-1)} w(t) d t\right)^{1 / p}=C[f]_{q, w}^{q / p}=1
$$

and $g_{1}$ is decreasing because $\frac{f^{o}}{w}$ is by the properties of $f^{o}$. Moreover, for each maximal level interval $A$ of $f$,

$$
\int_{A} f(t) g_{1}(t) d t=\int_{A} f^{o}(t) g_{1}(t) d t
$$

and so

$$
\int f(t) g_{1}(t) d t=\int f^{o}(t) g_{1}(t) d t=C \int\left(\frac{f^{o}(t)}{w(t)}\right)^{q-1} f^{o}(t) d t=[f]_{q, w},
$$

which proves (12).

From (12) we immediately obtain a D-type Hölder-Rogers inequality

$$
\int_{I} f(t) g(t) d t \leqslant[f]_{q, w}\|g\|_{L_{p}(w)} \quad \text { for an arbitrary } f \geqslant 0 \quad \text { and any } 0 \leqslant g \downarrow .
$$




\section{The dual space of an Orlicz-Lorentz space}

By $\varphi$ we denote a Young function, that is, $\varphi:[0, \infty) \rightarrow[0, \infty], \varphi(0)=0$, and $\varphi$ is convex. Let $\psi$ be the function complementary to $\varphi$, that is, $\psi(s)=\sup _{t \geqslant 0}\{s t-\varphi(t)\}, s \geqslant 0$. A finite-valued Young function is called an Orlicz function, and an Orlicz function $\varphi$ which is 0 only at 0 and such that $\lim _{t \rightarrow 0+} \varphi(t) / t=\lim _{t \rightarrow \infty} t / \varphi(t)=0$ is called an $N$-function. It is well known that $\psi$ is an $N$-function whenever $\varphi$ is $[25,39]$.

For a Young function $\varphi$ and $w \in \mathcal{W}_{d}(I)$, the Orlicz-Lorentz space is the set

$$
\Lambda_{\varphi, w}=\left\{f \in L^{0}: \exists_{\lambda>0} I_{\varphi, w}(\lambda f)<\infty\right\},
$$

where $I_{\varphi, w}(f)=\int_{I} \varphi\left(f^{*}(t)\right) w(t) d t$; it is equipped either with the Luxemburg-Nakano norm

$$
\|f\|_{\Lambda_{\varphi, w}}=\inf \left\{\varepsilon>0: I_{\varphi, w}(f / \varepsilon) \leqslant 1\right\},
$$

or with the Orlicz norm in the Amemiya form

$$
\|f\|_{\Lambda_{\varphi, w}}^{0}=\inf _{k>0} \frac{1}{k}\left(1+I_{\varphi, w}(k f)\right) .
$$

By $\Lambda_{\varphi, w}$ we denote the Orlicz-Lorentz space equipped with the Luxemburg-Nakano norm $\|\cdot\|_{\Lambda_{\varphi, w}}$, and by $\Lambda_{\varphi, w}^{0}$ this same space equipped with the Orlicz (Amemiya) norm $\|\cdot\|_{\Lambda_{\varphi, w}}^{0}$.

Kamińska and Raynaud in [23] defined the spaces $\mathcal{M}_{\varphi, w}$ : For a strictly increasing Orlicz function $\varphi$ and a decreasing weight $w$, let

$$
\mathcal{M}_{\varphi, w}=\left\{f \in L^{0}: \mathcal{P}_{\varphi, w}(f / \lambda)<\infty \text { for some } \lambda>0\right\},
$$

where the modular $\mathcal{P}_{\varphi, w}$ is defined as

$$
\mathcal{P}_{\varphi, w}(f)=\inf \left\{\int_{I} \varphi\left(\frac{f^{*}}{|g|}\right)|g|: g<^{*} w\right\} .
$$

The following duality theorem was proved in [19].

5.1. Theorem. Let $\varphi$ be an $N$-function and $w \in \mathcal{W}_{d}$. Then the Köthe dual spaces to the Orlicz-Lorentz spaces $\Lambda_{\varphi, w}$ and $\Lambda_{\varphi, w}^{0}$ are expressed as

$$
\left(\Lambda_{\varphi, w}\right)^{\prime}=\mathcal{M}_{\psi, w}^{0} \text { and }\left(\Lambda_{\varphi, w}^{0}\right)^{\prime}=\mathcal{M}_{\psi, w} \text {, }
$$

with equality of norms

$$
\begin{aligned}
& \|f\|_{\left(\Lambda_{\varphi, w}\right)^{\prime}}=\|f\|_{\mathcal{M}_{\psi, w}^{0}}=\inf _{k>0}\left\{\frac{\mathcal{P}_{\psi, w}(k f)+1}{k}\right\}, \\
& \|f\|_{\left(\Lambda_{\varphi, w}^{0}\right)^{\prime}}=\|f\|_{\mathcal{M}_{\psi, w}}=\inf \left\{\lambda>0: \mathcal{P}_{\psi, w}\left(\frac{f}{\lambda}\right) \leqslant 1\right\},
\end{aligned}
$$


where

$$
\mathcal{P}_{\psi, w}(f)=\inf \left\{\int_{I} \psi\left(\frac{f^{*}(t)}{|g(t)|}\right)|g(t)| d t: g<w\right\} .
$$

If, in addition, we assume that $W(\infty)=\infty$ for $I=[0, \infty)$, then we also have that

$$
\mathcal{P}_{\psi, w}(f)=\int_{I} \psi\left(\frac{\left(f^{*}\right)^{o}(t)}{w(t)}\right) w(t) d t=\int_{I} \psi\left(\frac{f^{*}(t)}{w^{f^{*}}(t)}\right) w^{f^{*}}(t) d t
$$

where $\left(f^{*}\right)^{o}$ is the level functions of $f^{*}$ with respect to $w$, and $w^{f^{*}}$ is the inverse level function of $w$ with respect to $f^{*}$.

We will extend this result to all Young functions and all decreasing weights, using Sinnamon's results. Notice that the same characterization may be found in Nakamura's paper [40] for an $N$-function $\varphi$.

5.2. Theorem. Let $\varphi$ be a Young function and $w \in \mathcal{W}_{d}$. Then the Köthe dual spaces to the Orlicz-Lorentz spaces $\Lambda_{\varphi, w}$ and $\Lambda_{\varphi, w}^{0}$ are expressed as

$$
\left(\Lambda_{\varphi, w}\right)^{\prime}=\mathcal{M}_{\psi, w}^{0} \text { and }\left(\Lambda_{\varphi, w}^{0}\right)^{\prime}=\mathcal{M}_{\psi, w},
$$

where this time the $\mathcal{M}_{\psi, w}$ spaces are defined with the use of the modular

$$
P_{\psi, w}(f)=\int_{I} \psi\left(\frac{\left(f^{*}\right)^{o}(t)}{w(t)}\right) w(t) d t=\int_{I} \psi\left(\left(\frac{f^{*}}{w}\right)^{s}(t)\right) w(t) d t
$$

5.3. Remark. We will regard the $\mathcal{M}_{\psi, w}$ spaces as duals to $\Lambda_{\varphi, w}$, and hence we have replaced the modular $\mathcal{P}_{\psi, w}$ by the modular $P_{\psi, w}$ in the definition. Of course, under the assumptions of Theorem 5.1, they are equal.

Proof of Theorem 5.2. Let $L_{\psi}(w)$ and $L_{\psi}^{0}(w)$ be an Orlicz spaces over the measure $d \mu=$ $w(t) d t$, equipped with the Luxemburg-Nakano norm and the Amemiya norm, respectively. For a decreasing function $g \in\left(\Lambda_{\varphi, w}\right)^{\prime}$, we have

$$
\|g\|_{\left(\Lambda_{\varphi, w}\right)^{\prime}}=\sup _{\|f\|_{\Lambda_{\varphi, w}} \leqslant 1} \int f(t) g(t) d t=\sup _{\substack{f \downarrow \\ f f \|_{L_{\varphi}(w)} \leqslant 1}} \int f(t) \frac{g(t)}{w(t)} w(t) d t=\left\|\frac{g}{w}\right\|_{D\left(L_{\psi}^{0}(w)\right)},
$$

where $\left\|\frac{g}{w}\right\|_{D\left(L_{\psi}^{0}(w)\right)}$ denotes the down norm in the Orlicz space $L_{\psi}^{0}(w)$. The last equality holds for all Young functions, which follows from a result by Hudzik and Maligranda [16]. Applying Sinnamon's Theorem 3.2 and the relationship between Halperin's and Sinnamon's level functions, we get

$$
\left\|\frac{g}{w}\right\|_{D\left(L_{\psi}^{0}(w)\right)}=\left\|\left(\frac{g}{w}\right)^{s}\right\|_{L_{\psi}^{0}(w)}=\left\|\frac{g^{o}}{w}\right\|_{L_{\psi}^{0}(w)} .
$$


Moreover,

$$
\left\|\frac{g^{o}}{w}\right\|_{L_{\psi}^{0}(w)}=\inf _{k>0}\left\{\frac{\int_{I} \psi\left(k \frac{g^{o}(t)}{w(t)}\right) w(t) d t+1}{k}\right\}=\|g\|_{\mathcal{M}_{\psi, w}^{0}} .
$$

This establishes the desired formula.

\section{Applications to geometry}

Convexity and smoothness (Gateaux smoothness) are the basic geometrical properties in Banach space theory, and they are dual to each other. It is well known when classical spaces such as the Lebesgue or Orlicz space enjoy these properties, and it is a rather commonplace situation. Therefore, one may be surprised to learn that Lorentz spaces and Orlicz-Lorentz spaces are essentially never smooth, unless the weight is constant. The following two-dimensional example of the Lorentz space $\lambda_{p, w}^{(2)}$ provides an intuitive understanding why it has to be so.

6.1. Example. Consider $\lambda_{p, w}^{(2)}$, where $w=\left(w_{1}, w_{2}\right)$ satisfies $w_{1}>w_{2}>0$ (if $w_{1}=w_{2}$, then we have just the space $\left.l^{p}\right)$. The norm on $\lambda_{p, w}^{(2)}$ may be written as

$$
\|x\|_{\lambda_{p, w}^{(2)}}=\left(\left(x_{1}^{*}\right)^{p} w_{1}+\left(x_{2}^{*}\right)^{p} w_{2}\right)^{1 / p}=\max \left(\left|x_{1}\right|^{p} w_{1}+\left|x_{2}\right|^{p} w_{2},\left|x_{2}\right|^{p} w_{1}+\left|x_{1}\right|^{p} w_{2}\right)^{1 / p} .
$$

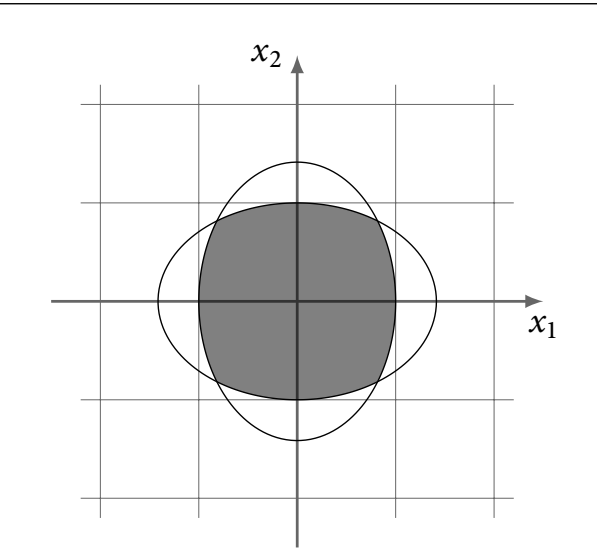

Figure 3. The unit ball in $\lambda_{2, w}^{(2)}$ for $w=\left(1, \frac{1}{2}\right)$ 
Geometrically, this means that the unit ball in $\lambda_{p, w}^{(2)}$ is just an intersection of the unit balls in two weighted $l^{p}$-spaces, i.e. one with the weight $\left(w_{1}, w_{2}\right)$ and the other with the weight $\left(w_{2}, w_{1}\right)$. One can see then that this ball is not smooth on the line $x_{1}=x_{2}$.

The full description of Gateaux smooth points of the unit sphere in Orlicz-Lorentz spaces is already known when the weight $w$ is strictly decreasing and $\varphi$ is a differentiable Orlicz function with $\varphi>0$, thanks to the work by Cuenya and Levis [33,34]. Moreover, in [2] a description of all extreme points of the unit sphere in the dual of $\Lambda_{p, w}$ was given - also under the assumption that $w$ is strictly decreasing. We are not going to give such a special theorem, but we will rather show that for all Young functions $\varphi$ and non-constant weights $w$ the spaces $\mathcal{M}_{\varphi, w}$ are not strictly convex. The single assumption, i.e. that the weight $w$ is not equal to a constant on the whole $I$, is necessary because otherwise the Orlicz-Lorentz space would reduce to the respective Orlicz space.

6.2. Definition. We say that $x \in S(X)$ (where $S(X)$ is the unit sphere in the Banach space $X)$ is a rotund point if $y \in S(X)$ and $\left\|\frac{x+y}{2}\right\|_{X}=1$ imply that $x=y$, or, in other words, if there is no interval in $S(X)$ such that $x$ is one of its end points.

We start with the following lemma.

6.3. Lemma. Let $\varphi$ be a Young function and $w \in \mathcal{W}_{d}$. If $f=f^{*} \in S\left(\mathcal{M}_{\varphi, w}\right)\left[\operatorname{or} f=f^{*} \in\right.$ $\left.S\left(\mathcal{M}_{\varphi, w}^{0}\right)\right]$ is such that $f \neq f^{0}$, then $f$ is not a rotund point of $S\left(\mathcal{M}_{\varphi, w}\right)\left[\right.$ of $\left.S\left(\mathcal{M}_{\varphi, w}^{0}\right)\right]$.

Proof. Let $f=f^{*} \in S\left(\mathcal{M}_{\varphi, w}\right)$ be such that $f \neq f^{o}$. This means that there is a maximal level interval $(a, b)$ of $f$ with respect to $w$ such that $f^{o}=R_{f}(a, b) w \neq f$ on $(a, b)$, where $R_{f}(a, b)=F(a, b) / W(a, b)$. In particular,

$$
R_{f}(a, t) \leqslant R_{f}(a, b) \text { for } t \in(a, b) .
$$

Then $\left(f+f^{o}\right)^{*}=f+f^{o}$, and the function $h=\frac{f+f^{o}}{2}$ is decreasing. Moreover, $(a, b)$, as any maximal level interval of $f$, is also a maximal level interval of $h$ because

$$
R_{h}(a, t) \leqslant R_{f}(a, t)
$$

while $R_{h}(a, b)=R_{f}(a, b)$. Hence also $h^{o}=f^{o}$. Therefore, $h \in S\left(\mathcal{M}_{\varphi, w}\right)$ just as $f^{o} \in$ $S\left(\mathcal{M}_{\varphi, w}\right)$ and, since $f \neq f^{o}$, we conclude that $f$ is not a rotund point.

6.4. Theorem. Let $\varphi$ be a Young function and let $w \in \mathcal{W}_{d}$ be a non-constant weight function. Then neither $\mathcal{M}_{\varphi, w}$ nor $\mathcal{M}_{\varphi, w}^{0}$ is strictly convex. 
Proof. We need to show that under our assumption on the weight $w$ there is $f=f^{*} \epsilon$ $S\left(\mathcal{M}_{\varphi, w}\right)$ satisfying the assumptions of the above lemma. Let $(a, b)$ be an interval such that $0<a<b<\infty$, and let $w$ be non-constant on $(a, b)$. Putting

$$
f=w \chi_{(0, a)}+\frac{W(a, b)}{b-a} \chi_{(a, b)}
$$

we have $f=f^{*}$ and

$$
R_{f}(a, t)=\frac{W(a, b)(t-a)}{W(a, t)(b-a)}<1=R_{f}(a, b),
$$

since $\frac{W(a, b)}{b-a}<\frac{W(a, t)}{t-a}$ for all $t \in(a, b)$. The above implies that

$$
R_{f}(0, t) \leqslant 1=R_{f}(0, b)
$$

for all $t \in(0, b)$. In consequence, $(0, b)$ is a maximal level interval of $f$, and $f^{o}=w \chi_{(0, b)} \neq$ $f$. This is the desired conclusion.

To derive the smoothness of Orlicz-Lorentz spaces, we will discuss the order continuity of the $\mathcal{M}_{\varphi, w}$ spaces. In the case of regular weights smoothness can be concluded from Corollary 5.2 in [22]. Let us show that the regularity assumption may be omitted. We start with a technical result concerning the level functions.

6.5. Lemma. Let $\varphi$ be a Young function and let $w \in \mathcal{W}_{d}$. If $0 \leqslant f_{n} \leqslant f=f^{*} \in \mathcal{M}_{\varphi, w}$, with $\varphi>0$ in the case when $I=[0, \infty)$, are such that $f_{n}=f_{n}^{*},\left|\operatorname{supp} f_{n}\right|<\infty$, and $f_{n}(t) \rightarrow 0$ for every $t>0$, then $f_{n}^{o}(t) \rightarrow 0$ for every $t>0$.

Proof. Suppose $I=[0, \infty)$ and $W(\infty)=\infty$. We may assume that $P_{\varphi, w}(f)<\infty$. Fix $s>0$. If $f_{n}(s)=f_{n}^{o}(s)$ for all but a finite number of $n$ 's, then the claim follows directly from the assumption. Suppose therefore that $s \in\left(a_{n}, b_{n}\right)$ for all $n$, where each $\left(a_{n}, b_{n}\right)$ is a maximal level interval of the respective $f_{n}$. Then $f_{n}^{o}(s)=R_{f_{n}}\left(a_{n}, b_{n}\right) w(s)$, and we need to show that $R_{f_{n}}\left(a_{n}, b_{n}\right) \rightarrow 0$ as $n \rightarrow \infty$. Let $\varepsilon>0$ be arbitrary. We have

$$
P_{\varphi, w}(f) \geqslant P_{\varphi, w}\left(f_{n}\right)=\int_{0}^{\infty} \varphi\left(\frac{f_{n}^{o}(t)}{w(t)}\right) w(t) d t \geqslant \int_{a_{n}}^{b_{n}} \varphi\left(R_{f_{n}}\left(a_{n}, b_{n}\right)\right) w(t) d t,
$$

hence

$$
R_{f_{n}}\left(a_{n}, b_{n}\right) \leqslant \varphi^{-1}\left(\frac{P_{\varphi, w}(f)}{W\left(s, b_{n}\right)}\right) .
$$

Since $W(\infty)=\infty$, there is $M>0$ such that if $b_{n}>M$ then $R_{f_{n}}\left(a_{n}, b_{n}\right)<\varepsilon$. Now, for $b_{n} \leqslant M$, we have two possibilities: either $a_{n}<s / 2$ or $s / 2<a_{n}<s$. In the first case,

$$
R_{f_{n}}\left(a_{n}, b_{n}\right) \leqslant \frac{\int_{0}^{M} f_{n}(t) d t}{W(s / 2, s)} \rightarrow 0 \quad \text { as } n \rightarrow \infty .
$$


In the other case we estimate as follows

$$
R_{f_{n}}\left(a_{n}, b_{n}\right) \leqslant \frac{\int_{a_{n}}^{b_{n}} f_{n}(t) d t}{w(M)\left(b_{n}-a_{n}\right)} \leqslant \frac{\left(b_{n}-a_{n}\right) f_{n}(s / 2)}{w(M)\left(b_{n}-a_{n}\right)}=\frac{f_{n}(s / 2)}{w(M)} \rightarrow 0 \quad \text { as } n \rightarrow \infty .
$$

We conclude that if $n$ is large enough, then $R_{f_{n}}\left(a_{n}, b_{n}\right)<\varepsilon$.

When $W(\infty)<\infty$, it is known that $L^{\infty} \subset \Lambda_{\psi, w}$ and, by duality, $\mathcal{M}_{\varphi, w} \subset L^{1}$. Therefore $\int_{0}^{\infty} f(t) d t \geqslant \int_{0}^{\infty} f_{n}(t) d t \rightarrow 0$. In consequence, if $b_{n}>2 s$, then

$$
R_{f_{n}}\left(a_{n}, b_{n}\right) \leqslant \frac{\int_{0}^{\infty} f_{n}(t) d t}{W(s, 2 s)} \rightarrow 0 \quad \text { as } n \rightarrow \infty,
$$

while if $b_{n} \leqslant 2 s$ we proceed as in the first part. The case when $I=[0,1]$ is simpler and the proof will be omitted.

We need one more observation.

6.6. Lemma. Let $I=[0, \infty)$, and let $\varphi$ be a Young function and $w \in \mathcal{W}_{d}$. If $\varphi \in \Delta_{2}$ and $f \in \mathcal{M}_{\varphi, w}$, then $f^{*}(\infty)=0$.

Proof. Suppose there is $f \in \mathcal{M}_{\varphi, w}$ with $f^{*}(\infty)>0$. Then also $\chi_{[0, \infty)} \in \mathcal{M}_{\varphi, w}$. This means that $L^{\infty} \subset \mathcal{M}_{\varphi, w}$ and, by duality, that $\Lambda_{\psi, w} \subset L^{1}$. But the latter may occur only when $w(\infty)>0$ and $\psi$ for small arguments is equivalent to a linear function. In such a case, however, complementary function to $\psi$ is $\varphi$ and it is equal to 0 on some interval $[0, b]$, which contradicts the assumption that $\varphi \in \Delta_{2}$ (cf. [39]).

6.7. Theorem. Let $\varphi$ be a Young function and let $w \in \mathcal{W}_{d}$. If $\varphi \in \Delta_{2}$ in the case when $I=[0, \infty)$ or $\varphi \in \Delta_{2}(\infty)$ in the case when $I=[0,1]$, then $\mathcal{M}_{\varphi, w}$ is order continuous.

Proof. Let $I=[0, \infty)$ and let $0 \leqslant g_{n} \leqslant g \in \mathcal{M}_{\varphi, w}$ be such that $g_{n} \rightarrow 0$ almost everywhere as $n \rightarrow \infty$. We have to show that $\left\|g_{n}\right\|_{\mathcal{M}_{\varphi, w}} \rightarrow 0$ as $n \rightarrow \infty$, and we only need to consider the case when $\left|\operatorname{supp} g_{n}\right|<\infty$ for each $n$ thanks to the Fatou property of $\mathcal{M}_{\varphi, w}$. If there were $0 \leqslant g_{n} \leqslant g \in \mathcal{M}_{\varphi, w}$ with $\left\|g_{n}\right\|_{\mathcal{M}_{\varphi, w}}>\delta>0$, then we could find another sequence $\left(h_{n}\right)$ such that $\left|\operatorname{supp} h_{n}\right|<\infty, 0 \leqslant h_{n} \leqslant g_{n}$, and $\left\|h_{n}\right\|_{\mathcal{M}_{\varphi, w}}>\delta / 2>0$.

From Lemma 6.6 we know that $\varphi \in \Delta_{2}$ and $g \in \mathcal{M}_{\varphi, w}$ imply $g^{*}(\infty)=0$. Put $f_{n}:=g_{n}^{*}$ and $f:=g^{*}$. Then $f_{n}(t) \rightarrow 0$ for all $t>0$. By Lemma 6.5 , we also have $f_{n}^{o}(t) \rightarrow 0$ for all $t>0$. But $\varphi \in \Delta_{2}$ implies that $P_{\varphi, w}(\lambda f)<\infty$ for every $\lambda>0$. Moreover,

$$
P_{\varphi, w}\left(\lambda f_{n}\right)=\int_{0}^{\infty} \varphi\left(\frac{\lambda f_{n}^{o}(t)}{w(t)}\right) w(t) d t \rightarrow 0 \quad \text { as } n \rightarrow \infty
$$

for every $\lambda>0$. This means that for arbitrarily large $\lambda>0$ we can find $N$ such that for all $n>N$ we have $P_{\varphi, w}\left(\lambda f_{n}\right) \leqslant 1$, i.e. $\left\|g_{n}\right\|_{\mathcal{M}_{\varphi, w}} \leqslant 1 / \lambda$. From the arbitrariness of $\lambda>0$, $\left\|g_{n}\right\|_{\mathcal{M}_{\varphi, w}} \rightarrow 0$ as $n \rightarrow \infty$, which is our claim. 
6.8. Corollary. Let $\varphi$ be a Young function and let $w \in \mathcal{W}_{d}$ be a non-constant weight. If $\varphi \in \Delta_{2}$ in the case when $I=[0, \infty)$ or $\varphi \in \Delta_{2}(\infty)$ in the case when $I=[0,1]$, then neither $\Lambda_{\psi, w}$ nor $\Lambda_{\psi, w}^{0}$ is Gateaux smooth.

Proof. Under our assumptions, by Theorems 5.2 and 6.7, the space $\Lambda_{\psi, w}^{0}$ is the dual of $\mathcal{M}_{\varphi, w}$ which is not strictly convex, by Theorem 6.4. Therefore, $\Lambda_{\psi, w}^{0}$ is not Gateaux smooth. Similarly for $\Lambda_{\psi, w}$.

6.9. Remark. We did not need to make the $\Delta_{2}$ assumption above. It would be enough to ensure that all simple functions in $\mathcal{M}_{\varphi, w}$ are order continuous because then $\Lambda_{\psi, w}^{0}=$ $\left[\left(\mathcal{M}_{\varphi, w}\right)_{a}\right]^{*}$, where $\left(\mathcal{M}_{\varphi, w}\right)_{a}$ is the subspace of the order continuous elements of $\mathcal{M}_{\varphi, w}$.

Acknowledgements. The first author has been partially supported by the National Science Centre (NCN), Poland, Grant No. DEC-2012/07/B/ST1/03360. The second author has been partially supported by the grant $04 / 43 / \mathrm{DSPB} / 0086$ from the Polish Ministry of Science and Higher Education. The second author wishes to thank Professor Yves Raynaud for inspiring conversations on the subject. All the authors are also grateful to Dr. Michał Goliński and Dr. Paweł Mleczko for assistance in making the plots.

\section{References}

[1] C. Bennett and R. Sharpley, Interpolation of Operators, Academic Press, Boston 1988.

[2] V. A. Biktasheva, Level functions and extreme points of the space $M_{\omega}^{q}(1<q<\infty)$, Mat. Zametki 35 (1984), no. 1, 9-18.

[3] D. W. Boyd, The Hilbert transform on rearrangement-invariant spaces, Canad. J. Math. 19 (1967), 599-616.

[4] M. J. Carro and J. Soria, Weighted Lorentz spaces and the Hardy operator, J. Funct. Anal. 112 (1993), no. 2, 480-494, DOI 10.1006/jfan.1993.1042.

[5] M. J. Carro and J. Soria, Boundedness of some integral operators, Canad. J. Math. 45 (1993), no. 6, 1155-1166, DOI 10.4153/CJM-1993-064-2.

[6] S. Chen, Y. Cui, H. Hudzik, and T. Wang, On some solved and unsolved problems in geometry of certain classes of Banach function spaces, Unsolved Problems on Mathematics for the 21st Century, IOS, Amsterdam, 2001.

[7] M. Cwikel, A. Kamińska, L. Maligranda, and L. Pick, Are generalized Lorentz "spaces" really spaces?, Proc. Amer. Math. Soc. 132 (2004), no. 12, 3615-3625, DOI 10.1090/S0002-9939-04-07477-5.

[8] P. Foralewski, H. Hudzik, and P. Kolwicz, Non-squareness properties of Orlicz-Lorentz function spaces, J. Inequal. Appl. 32 (2013), 25.

[9] P. Foralewski, H. Hudzik, and P. Kolwicz, Non-squareness properties of Orlicz-Lorentz sequence spaces, J. Funct. Anal. 264 (2013), no. 2, 605-629, DOI 10.1186/1029-242X-2013-32.

[10] A. Gogatishvili and L. Pick, Duality principles and reduction theorems, Math. Inequal. Appl. 3 (2000), no. 4, 539-558, DOI 10.7153/mia-03-51.

[11] A. Haaker, On the conjugate space of the Lorentz space, Technical Report, Lund 1970, 1-23.

[12] I. Halperin, Function spaces, Canad. J. Math. 5 (1953), 273-288. 
[13] H. P. Heinig and A. Kufner, Hardy operators of monotone functions and sequences in Orlicz spaces, J. London Math. Soc. (2) 53 (1996), 256-270, DOI 10.1112/jlms/53.2.256.

[14] H. P. Heinig and L. Maligranda, Weighted inequalities for monotone and concave functions, Studia Math. 116 (1995), no. 2, 133-165.

[15] H. Hudzik, A. Kamińska, and M. Mastyło, On the duals of Orlicz-Lorentz space, Proc. Amer. Math. Soc. 130 (2002), no. 6, 1645-1654, DOI 10.1090/S0002-9939-02-05997-X.

[16] H. Hudzik and L. Maligranda, Amemiya norm equals Orlicz norm in general, Indag. Math. (N.S.) 11 (2000), no. 4, 573-585, DOI 10.1016/S0019-3577(00)80026-9.

[17] A. Kamińska, Some remarks on Orlicz-Lorentz spaces, Math. Nachr. 147 (1990), 29-38, DOI 10.1002/mana.19901470104.

[18] A. Kamińska and D. Kubiak, On the dual of Cesáro function space, Nonlinear Anal. 75 (2012), no. 5, 2760-2773, DOI 10.1016/j.na.2011.11.019.

[19] A. Kamińska, K. Leśnik, and Y. Raynaud, Dual spaces to Orlicz-Lorentz spaces, Studia Math. 222 (2014), no. 3, 229-261, DOI 10.4064/sm222-3-3.

[20] A. Kamińska and L. Maligranda, Order convexity and concavity in Lorentz spaces $\Lambda_{p, w}, 0<p<\infty$, Studia Math. 160 (2004), no. 3, 267-286, DOI 10.4064/sm160-3-5.

[21] A. Kamińska and M. Mastyło, Abstract duality Sawyer formula and its applications, Monatsh. Math. 151 (2007), no. 3, 223-245, DOI 10.1007/s00605-007-0445-9.

[22] A. Kamińska and Y. Raynaud, Isomorphic copies in the lattice $E$ and its symmetrization $E^{(*)}$ with applications to Orlicz-Lorentz spaces, J. Funct. Anal. 257 (2009), no. 1, 271-331, DOI 10.1016/j.jfa.2009.02.016.

[23] A. Kamińska and Y. Raynaud, New formulas for decreasing rearrangements and a class of Orlicz-Lorentz spaces, Rev. Mat. Complut. 27 (2014), no. 2, 587-621, DOI 10.1007/s13163-013-0119-1.

[24] R. Kerman, M. Milman, and G. Sinnamon, On the Brudnyi-Krugljak duality theory of spaces formed by the K-method of interpolation, Rev. Mat. Complut. 20 (2007), no. 2, 367-389, DOI 10.5209/rev_REMA.2007.v20.n2.16492.

[25] M. A. Krasnoselski 1 and Ya. B. Ruticki ${ }^{1}$, Convex Functions and Orlicz Spaces, P. Noordhoff Ltd., Groningen 1961.

[26] S. G. Krein, Yu. I. Petunin, and E. M. Semenov, Interpolation of Linear Operators, Amer. Math. Soc., Providence 1982.

[27] A. Kufner, L. Maligranda, and L.-E. Persson, The Hardy Inequality. About Its History and Some Related Results, Vydavatelsky Servis, Plzeň 2007.

[28] A. Kufner and L. E. Persson, Weighted Inequalities of Hardy Type, World Scientific, River Edge, NJ 2003, DOI 10.1142/5129.

[29] K. Leśnik, Monotone substochastic operators and a new Calderon couple, Studia Math. 227 (2015), no. 1, 21-39, DOI 10.4064/sm227-1-2.

[30] K. Leśnik and L. Maligranda, Abstract Cesàro spaces. Duality, J. Math. Anal. Appl. 424 (2015), no. 2, 932-951, DOI 10.1016/j.jmaa.2014.11.023.

[31] K. Leśnik and L. Maligranda, Abstract Cesàro spaces. Optimal range, Integral Equations Operator Theory 81 (2015), no. 2, 227-235, DOI 10.1007/s00020-014-2203-4.

[32] K. Leśnik and L. Maligranda, Interpolation of abstract Cesaro, Copson and Tandori spaces, Indag. Math. (N. S.) 27 (2016), no. 3, 764-785, DOI 10.1016/j.indag.2016.01.009.

[33] F. E. Levis and H. H. Cuenya, Gateaux differentiability for functional of type Orlicz-Lorentz, Acta Math. Univ. Comenian. (N.S.) 73 (2004), no. 1, 31-41.

[34] F. E. Levis and H. H. Cuenya, Gateaux differentiability in Orlicz-Lorentz spaces and applications, Math. Nachr. 280 (2007), no. 11, 1282-1296, DOI 10.1002/mana.200410553. 
[35] J. Lindenstrauss and L. Tzafriri, Classical Banach Spaces, II. Function Spaces, Springer-Verlag, Berlin-New York 1979.

[36] G. G. Lorentz, On the theory of spaces $\Lambda$, Pacific J. Math. 1 (1951), 411-429.

[37] G. G. Lorentz, Bernstein Polynomials, Univ. of Toronto Press, Toronto 1953.

[38] L. Maligranda, On Hardy's inequality in weighted rearrangement invariant spaces and applications. I, II, Proc. Amer. Math. Soc. 88 (1983), no. 1, 75-80, DOI 10.2307/2045111.

[39] L. Maligranda, Orlicz Spaces and Interpolation, Seminars in Math., vol. 5, University of Campinas, Campinas 1989.

[40] K. Nakamura, On $\Lambda(\phi, M)$-spaces, Bull. Fac. Sci. Ibaraki Univ. Ser. A 2 (1970), 31-39.

[41] E. Sawyer, Boundedness of classical operators on classical Lorentz spaces, Studia Math. 96 (1990), no. 2, 145-158.

[42] G. Sinnamon, Weighted Hardy and Opial-type inequalities, J. Math. Anal. Appl. 160 (1991), no. 2, 434-445, DOI 10.1016/0022-247X(91)90316-R.

[43] G. Sinnamon, Spaces defined by the level function and their duals, Studia Math. 111 (1994), no. 1, 19-52.

[44] G. Sinnamon, The level function in rearrangement invariant spaces, Publ. Mat. 45 (2001), no. 1, 175-198, DOI 10.5565/PUBLMAT_45101_08.

[45] G. Sinnamon, Transferring monotonicity in weighted norm inequalities, Collect. Math. 54 (2003), no. 2, $181-216$.

[46] G. Sinnamon, Monotonicity in Banach function spaces, Spring School (Prague, 2006), Nonlinear Analysis, Function Spaces and Applications, Vol. 8, Czech Academy of Sciences, Mathematical Institute, Praha, 2007, 205-240.

[47] A. Sparr, On the conjugate space of the Lorentz $L(\phi, q)$, Contemp. Math., vol. 445, Amer. Math. Soc., Providence, RI, 2007, 313-336, DOI 10.1090/conm/445/08610.

[48] V. D. Stepanov, The weighted Hardy's inequality for nonincreasing functions, Trans. Amer. Math. Soc. 338 (1993), 173-186, DOI 10.2307/2154450.

[49] A. C. Zaanen, Integration, North-Holland, Amsterdam 1967. 